\title{
Geoarqueología aplicada a la reconstrucción paleoambiental: La evolución del Holoceno superior en el NE de España
}

\author{
J.L. Peña Monné \\ Departamento de Geografía y Ordenación delTerritorio, Universidad de Zaragoza, \\ Pedro Cerbuna 12, E-50009 Zaragoza, España \\ jlpena@unizar.es
}

\begin{abstract}
RESUMEN
Los trabajos geoarqueológicos realizados en el NE de España se han centrado en el estudio de registros sedimentarios holocenos relacionados con áreas de ocupación que abarcan desde el Neolítico hasta Época Medieval y la Pequeña Edad del Hielo. El dinamismo de los procesos geomorfológicos en estos ambientes semiáridos permite obtener respuestas rápidas e identificables en forma de registros sedimentarios como consecuencia de pequeños cambios en la estabilidad de las laderas y en el funcionamiento de los sistemas fluviales. En este trabajo se sintetiza la información aportada por el análisis de laderas, abrigos rocosos, fondos de valle y conos aluviales. Se ha obtenido una sucesión de etapas evolutivas en cada uno de estos ambientes a partir de los cuales se intenta discriminar su génesis en relación con las fluctuaciones climáticas del Holoceno y con la intervención humana sobre el territorio. Los resultados de estos trabajos son de gran interés aplicado para la prospección arqueológica, para el conocimiento del poblamiento en épocas pasadas y para la reconstrucción paleoambiental en el NE de España, donde la fuerte erosión ha dificultado la conservación de numerosos yacimientos.
\end{abstract}

Palabras clave: Geoarqueología, Geomorfología, Holoceno, paleoambientes, zonas semiáridas.

\section{Geoarchaeology applied to paleo-environmental reconstruction: Late Holocene evolution in NE Spain}

\begin{abstract}
Geoarchaeological research carried out in NE Spain have focused on the study of Holocene geoarchaeological records linked with settlement areas from Neolithic to Middle Age and Little Ice Age. The dynamic of geomorphological processes, characteristic of these drylands, allow us to obtain fast and identifiable answers in the way of sedimentary records. These records are the consequence of slight changes in the slope stability and the operation of river systems. In this paper we present a synthesis of the information provided by slopes, rock shelters, valley bottoms, and alluvial fans. A succession of evolutionary stages was obtained on each environment identifying its origin according to Holocene climate fluctuations and human intervention over territory. The results of this research are of great interest when applied to archaeological prospection, the knowledge of past settlements, and the paleo-environmental reconstruction of NE Spain, where the strong erosion complicates the conservation of many archeological sites.
\end{abstract}

Key words: drylands, Geoarchaeology, Geomorphology, Holocene, paleo-environments.

ABRIDGED ENGLISH VERSION

\section{Introduction and methods}

Dryland environments in NE Spain have been at the centre of many geoarchaeological studies focused on the 
Peña Monné, J.L., 2018. Geoarqueología aplicada a la reconstrucción paleoambiental:... Boletín Geológico y Minero, 129 (1/2): $285-303$

use of sedimentary records and geomorphological features related to survey, territorial occupation, and paleo-environmental reconstruction of the Late Holocene. To this purpose, the complex relationships among settlements, erosion/accumulation processes, climate changes, and anthropic actions over landscape were analyzed. The objective was the establishment of Late Holocene evolutionary models, where the answers to various geomorphological environments, climates, and cultural stages are represented.

The aim of this paper is to synthesize the current data for this kind of geoarchaeological research from NE Spain (Fig. 1). Even though many available records exist (Fig. 2), our focus will be on three linked morphosedimentary environments: slopes, valleys and alluvial fans. Moreover, special attention will be paid to the evolution of rock shelters.

From the methodological point of view, the goal is to analyze the geoarchaeological records generated by those morphological environments to obtain answers to the aggradation or degradation phases, compared to the changes produced by the overcoming of geomorphological thresholds. Climatic fluctuations and human activities over a territory are triggers for these disruptions during the Later Holocene. The identification of the role of each of these points is the aim of this research.

\section{Results and discussion}

Slopes as an archaeological record

Slopes are the landforms with the fastest response to environmental changes. The oscillations between phases of stability and erosion are the most important record of the presence of changes in the slope dynamics, and they appeared as contrasts related with climate changes. Cold and wet stages tends to favour solifluction processes, the presence of vegetation, and even the formation of soils that contribute to slope stabilization; while during dry and warm phases incisions and destruction of the previous stable slopes are the rule.

Two stages of slope stabilization linked to climate changes were identified in NE Spain. The most important was generated, according to the existing research, since the Upper Chalcolithic (ca. 2300 BC) until the first Iron Age (ca. 800-650 AD). The slopes from this first moment are well preserved in high areas of the Iberian Ranges (Fig. 3) as well as in the Ebro Depression (Fig. 4.1). This phase of stability favours the development of a paleosol (Paleosol IIIA), which are only preserved in some points as are the Peña Enroque slopes (Fig. 5). This slope includes archaeological materials from the Bronze and Iron Ages. Its climate genesis is linked to the Subboreal and the cold phase of the Iron Age (Fig. 10). Since the Iberian Epoch a deep change in its dynamics was initiated. It is represented by big incisions and the development of gullies associated with the drier climate of the Subatlantic, but especially with Iberian and Roman anthropic activity (Fig. 4.2; 4.3). The second stage of regularization (Fig. 4.4) was during the LIA related with colder and wetter conditions (Fig. 10); it has two sub-stages (Fig. 5).

Rock shelters and their evolution

A special case in slope evolution is that of rock shelters. They are generated in geological formations with alternating layers with varying resistance to erosion. This situation favours the formation of caves or rock shelters that were useful for human settlements. In the middle Segre Valley (Lleida) it is possible to establish an evolutionary sequence starting with the formation of the rock shelters before and during the Bronze Age (Fig. 6a). After its human occupation they suffered a destruction process from the Iron Age epoch, with the loss of archaeological materials. The slopes gradually became regularized until the end of the Iron Age as it was previously set (Fig. 6b). Late incision permits us to see only partial records in different positions. The evolutionary process has to be reconstructed with these records (Fig. 6c).

Geoarchaeological information from valleys and alluvial fans

The secondary rivers, tributaries from lower Huerva river and from the central section of Ebro river, have important information about the changes on the fluvial dynamic during the Holocene. They are valleys excavated in the Miocene gypsum located in the central section of the Ebro Depression. They were infilled with sediments until their current flat aspect (they are named vales in the region). Many of these lateral valleys have pre-Holocene terrace levels, but the most important feature is the presence of thick Holocene accumulations, named the N3 level or principal level (Fig. 7). In some areas, the incision of this level permits the observation of their sedimentary sequences for study. The presence in the incision of two more Holocene nested terrace levels is also common (N2, N1) (Fig. 7). 
Peña Monné, J.L., 2018. Geoarqueología aplicada a la reconstrucción paleoambiental:... Boletín Geológico y Minero, 129 (1/2): $285-303$

The base of N3 terrace, dated around 7000-6000 BP (Neolithic), is composed of coarse detritic materials (sub-level A) followed by an ensemble from the Bronze and Iron Ages (sub-level B) and dated from ca. 3500 - 2500 BP. Archaeological materials are included in it. Finally sub-level $C$ represents the evolution of Iberian and Roman Epochs, followed by sub-level D corresponding to Late Roman (II-IV AD centuries) (Figs. 8.1, 8.4). These last two stages are composed of loamy sediments and in many cases they include archaeological structures, such as Roman villas. Due to its sedimentary thickness, granulometric composition, accumulation rate, and archaeological content it is a level formed as consequence of accelerated erosion produced by human intervention on the valley slopes due the fact that there is little evidence of calcareous gravels in the header basin over sub-levels $C$ and $D$.

Since 5th century up to the present day an incision phase started (Figs. 8.2, 10), with two interruptions. They gave place to the formation of N2 and N1 levels. They are also separated by one incision stage (Fig. 8.3). Both are related to a different dynamics than that of the N3 level, they are both the consequence of a climatic event that provided sediments from the limestone headwater basin located in the Muelas. According to the dates obtained these levels are consequence of the climate variability of the LIA (Fig. 10).

The outlets of these valleys to the main rivers gives way to the formation of alluvial fans. They have similar geoarchaeological records to the inner valleys (Figs. 9a, 9b). Even though the overlapping of the N2 stage over the N3 is very normal (Figs. 9a, 9c), they can also form independent alluvial fans (Fig. 9d).

\section{Conclusions}

In the drylands, which make up much of NE Spain, many archaeological sites have suffered significant modifications by erosion and accumulation processes. Therefore, geoarchaeological studies are really necessary to reconstruct their evolution.

The study of aggradation/incision stages in slopes, infilled valleys and alluvial fans, understood as a coupling system, permits the establishment of a joint evolution marked by stability and instability phases in slopes, accumulation and incision in valleys, and alluvial fans. Holocene climatic fluctuations are the cause of many of these processes, while others are favoured or even produced by human intervention. This is the case of slope incision phases since the lberian-Roman epoch until the present day, only interrupted by the climatic stabilization during LIA. N3 level formation in valleys and alluvial fans is also interpreted as consequence of that anthropic destabilization phase in the slopes, giving a thick geoarchaeological record. This record includes several features of archaeological sites which today have disappeared.

Ultimately, the construction of evolutionary models applied to different geomorphological environments, upon a geoarchaeological methodology is decisive for planning productive archaeological surveys and for completing the information about landscape evolution, settlement patterns, and resource exploitation in different epochs in the past.

\section{Introducción}

Desde finales de la década de 1970 se inició una línea de investigación en el Colegio Universitario de Teruel, proseguida en años posteriores en la Universidad de Zaragoza, centrada en la utilización de registros sedimentarios y elementos geomorfológicos relacionados con la prospección arqueológica y la ocupación humana del territorio. Estos estudios, surgidos de la colaboración de arqueólogos, geólogos y geógrafos, generaron algunos trabajos pioneros de clara identidad geoarqueológica, destacando el estudio de la evolución de laderas del cerro del castillo de Alfambra (Teruel) y áreas cercanas de la Sierra de Albarracín (Burillo et al., 1981a, 1981b, 1983, 1985b), del valle del río Segre (Lleida) (Peña-Monné, 1983a, 1983b; PeñaMonné et al., 1988) o del sector central del valle del Ebro (Burillo et al., 1985a; Pellicer et al., 1986; Sancho et al., 1988), así como algunos planteamientos meto- dológicos generales (Burillo and Peña-Monné, 1984a, 1984b, Burillo et al., 1985c). En estos estudios se siguen parcialmente las propuestas metodológicas de Vita-Finzi (1969), Bintliff (1976), van Zuidam (1975), Butzer (1980), Davidson (1980), Pope and van Andel (1984), Bruckner (1986), etc. para el ámbito mediterráneo, tomando como base de trabajo el registro geoarqueológico. Con este fin, se analizan las complejas relaciones entre asentamientos, procesos de erosión y acumulación, cambios climáticos y acción antrópica sobre el paisaje con vistas a intentar una reconstrucción paleoambiental para diferentes momentos evolutivos, centrándose de forma muy especial en el Holoceno superior (Burillo et al., 1986; Gutiérrez and Peña-Monné, 1992; Gutiérrez and Peña-Monné, 1998; González-Sampériz and Sopena, 2002, Peña-Monné et al., 2005b).

La trayectoria posterior de estos trabajos geoarqueológicos se ha enfocado al establecimiento de 
modelos evolutivos en diferentes ambientes geomorfológicos y a definir los tipos de respuesta que generan los cambios climáticos holocenos y la influencia antrópica sobre el territorio como consecuencia del aprovechamiento de sus recursos. En este trabajo se sintetizan estos aspectos geoarqueológicos y se ponen en relación con la evolución cultural prehistórica e histórica y con las fluctuaciones climáticas del Holoceno a escala mediterránea y global.

\section{Objetivos, área de estudio y metodología}

El objetivo principal de este trabajo es efectuar una recopilación de resultados de los diferentes estudios geoarqueológicos aplicados a la reconstrucción paleoambiental del Holoceno superior en el NE de España. Como objetivos particulares se van a analizar los datos procedentes de tres ambientes morfosedimentarios interconectados entre sí: las laderas, los valles y los conos aluviales, así como un aspecto más puntual de las laderas: la evolución de abrigos rocosos. La finalidad última es mostrar la relación existente entre la evolución de los ámbitos geomorfológicos propuestos y su conexión con cambios climáticos y procesos de ocupación humana.

El área de estudio abarca condiciones muy contrastadas desde el punto de vista topográfico, geológico y ambiental, ya que los ejemplos analizados se extienden desde los $160 \mathrm{~m}$ del fondo de la depresión del Ebro oriental a los $1600 \mathrm{~m}$ de altitud en la
Cordillera Ibérica, en ambientes que abarcan desde el semiárido hasta el subhúmedo (Fig. 1). Esta diversidad pretende mostrar coincidencias y diferencias en las respuestas geomorfológicas generadas por los factores climáticos y antrópicos y ayudar a dilucidar el papel de cada uno en el desencadenamiento de los procesos.

La combinación de factores en estos ambientes, con diferentes litologías, cubierta vegetal, orientación, pendientes, etc. genera paisajes de diferente sensibilidad ante los cambios, en los que la superación de determinados umbrales geomorfológicos provoca una reactivación dinámica más o menos rápida en forma de fases de agradación o degradación alternantes. Estas rupturas se transmiten entre los componentes del sistema formado por laderavalle-cono aluvial, ya que existe una interconexión funcional entre ellos que se manifiesta de forma más o menos evidente según el ambiente climático y permite establecer relaciones de contemporaneidad en su funcionamiento.

\section{Resultados y discusión}

Los registros geoarqueológicos principales que se han utilizado son los ya indicados: laderas con fases de acumulación más o menos alteradas por incisiones posteriores, abrigos rocosos sometidos a procesos evolutivos complejos y rellenos de valle en forma de terrazas escalonadas y encajadas reflejando fases

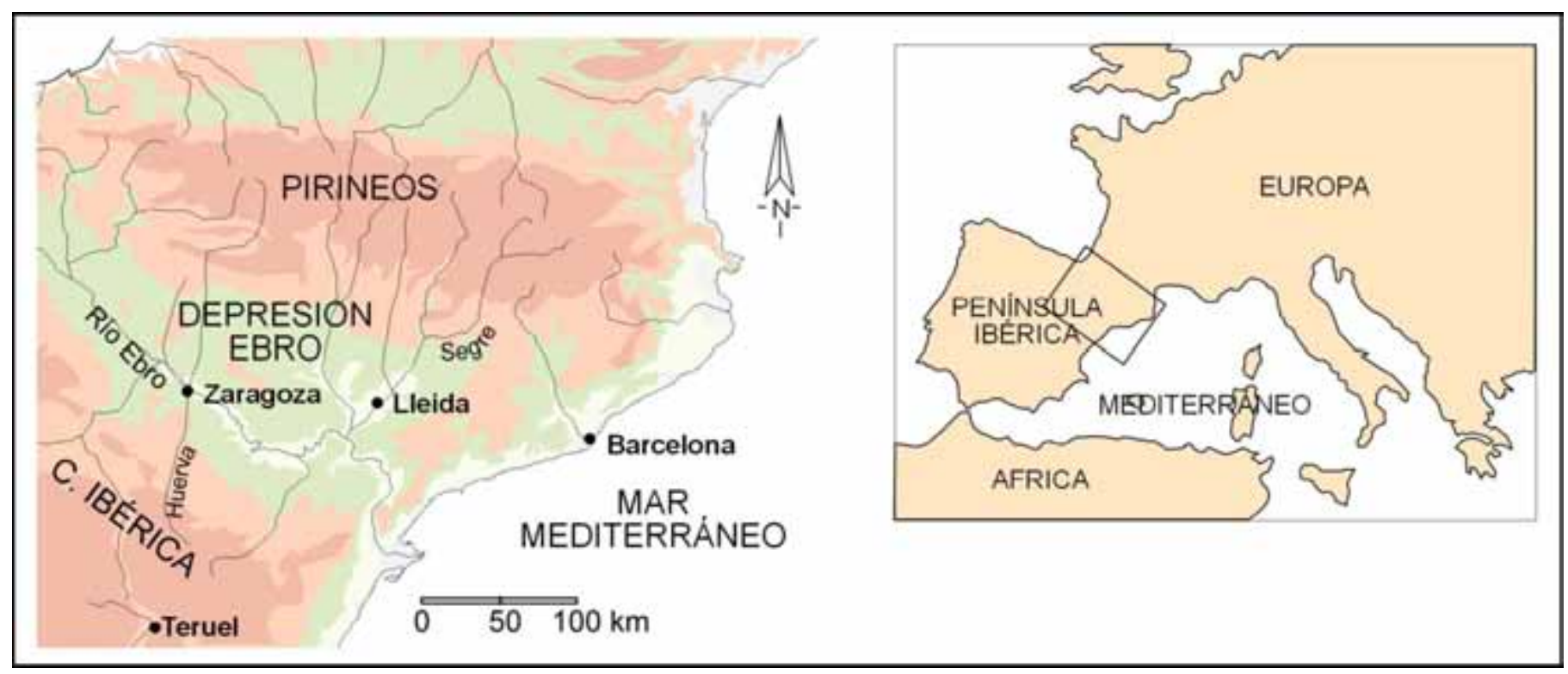

Figura 1. Mapa de situación del NE de España.

Figure 1. Location map of NE Spain. 
de agradación e incisión alternantes durante el Holoceno, que se prolongan hacia los cursos principales mediante conos aluviales. En la figura 2 se representan estos componentes del paisaje capaces de convertirse en registros geoarqueológicos, cuando hay en su entorno actividad humana intensa, caso de cuevas, abrigos, terrazas de cultivo, fortalezas, asentamientos dispersos de diferentes épocas y culturas y obras de defensa, que también generan por sí mismos registros útiles para la reconstrucción geoarqueológica, destacando especialmente los rellenos de los fosos defensivos en yacimientos desde la Edad del Hierro hasta época Medieval (Rubio et al., 2006; Peña-Monné et al., 2014a, 2014b).

\section{Las laderas como registro geoarqueológico}

Las laderas constituyen las formas de relieve con respuesta más rápida y específica ante los cambios generados en los factores que determinan su estabilidad. Su dinámica está definida por elementos internos, tales como litología, estructura geológica, permeabilidad, y externos como el clima, los suelos y la vegetación. El equilibrio entre estos factores se resuelve en la formación de un talud estable, en el que los procesos erosivos llegan a ser casi nulos. La pendiente entre las cornisas superiores y su nivel de base alcanza una situación de equilibrio favorecida por un clima apropiado para sostener un suelo y una cubierta vegetal protectora, adquiriendo una forma regularizada característica (Fig. 3). En términos generales son climas más fríos y húmedos que los actuales los más favorables para mantener laderas en estado de estabilización, mientras que los climas secos y cálidos favorecen la desestabilización de la ladera al no proporcionar las condiciones adecuadas para el mantenimiento de una cubierta vegetal continua y permitir que la escorrentía superficial acabe desembocando en un proceso creciente de erosión. La alter-

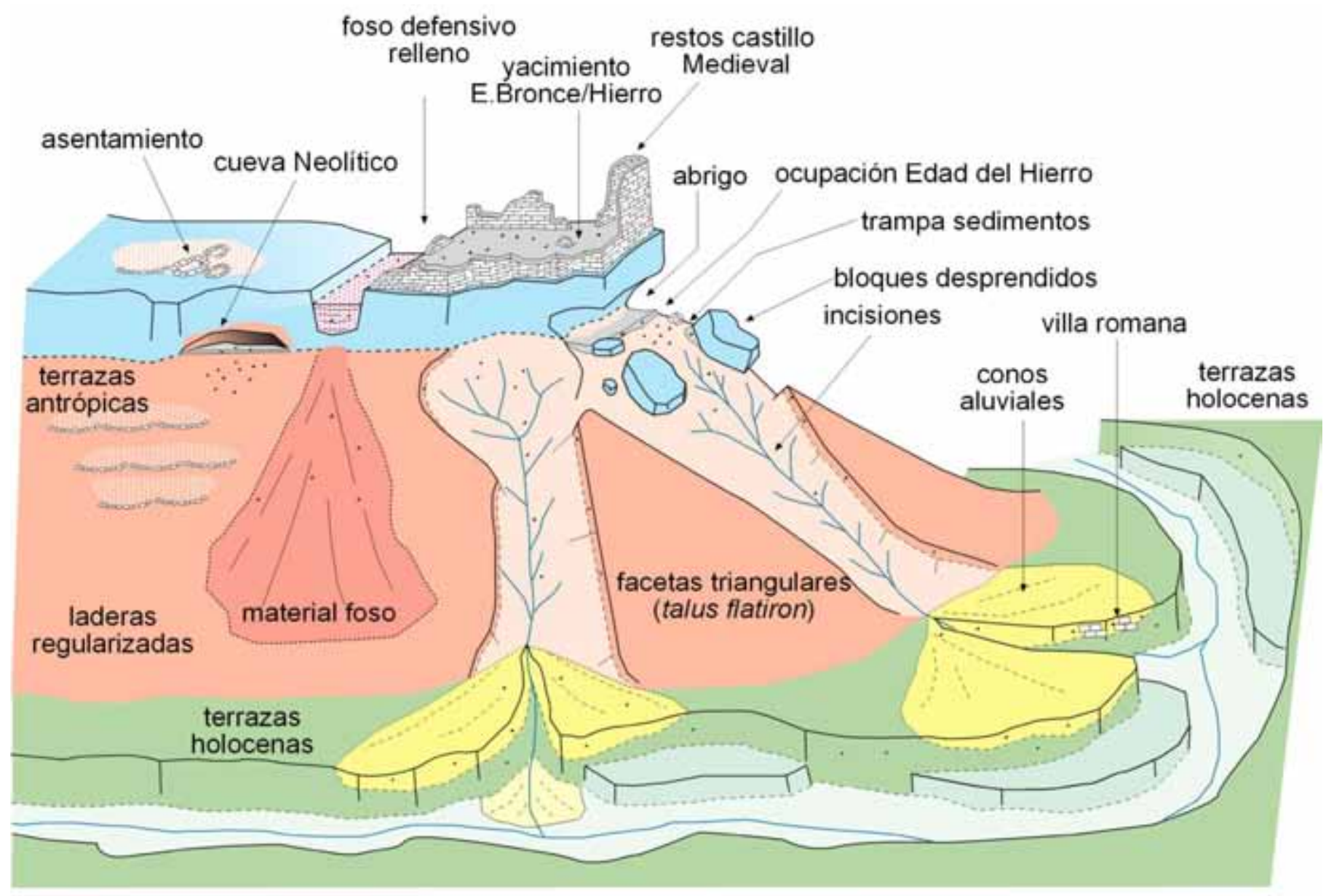

Figura 2. Representación esquemática de los diferentes tipos de registros geoarqueológicos más habituales en el NE de España. Figure 2. Schematic representation of the normally available different kinds of geoarchaeological records in NE Spain. 
nancia de fases de regularización e incisión se convierte así en un indicador de rupturas debidas a cambios climáticos; incluso las pequeñas fluctuaciones holocenas pueden generar estas situaciones contrastadas en la dinámica de laderas. Por otra parte, algunos de los factores externos también pueden ser modificados por la acción humana, como es el caso de la vegetación y los suelos, mediante sobrepastoreo, incendios, aprovechamiento de la madera, creación de áreas de cultivo, etc. determinando desestabilización brusca que genera procesos de incisión más intensos y rápidos que los causados por cambios
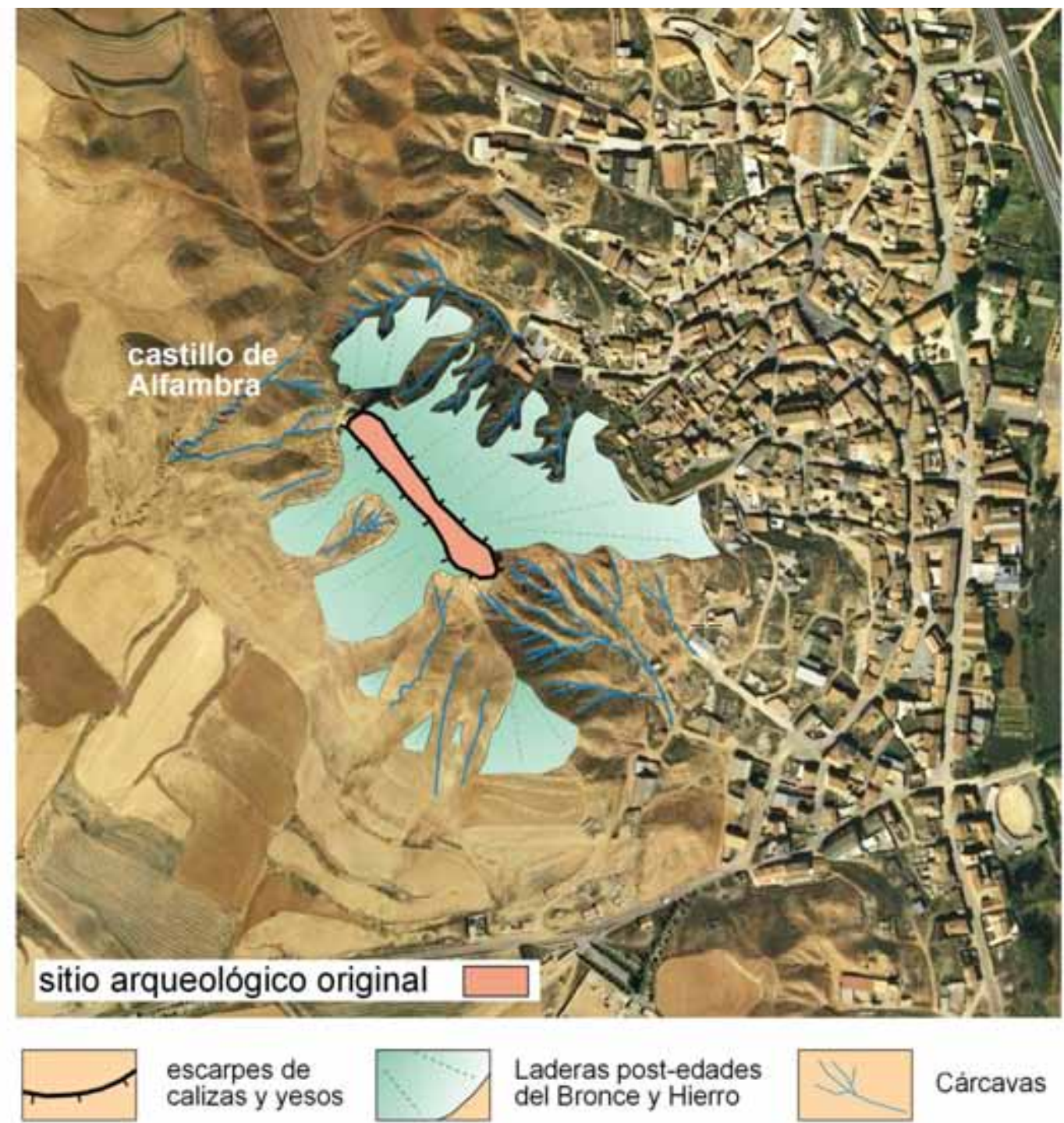

escarpes de calizas y yesos

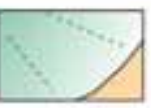

Laderas post-edades del Bronce y Hierro

Cárcavas

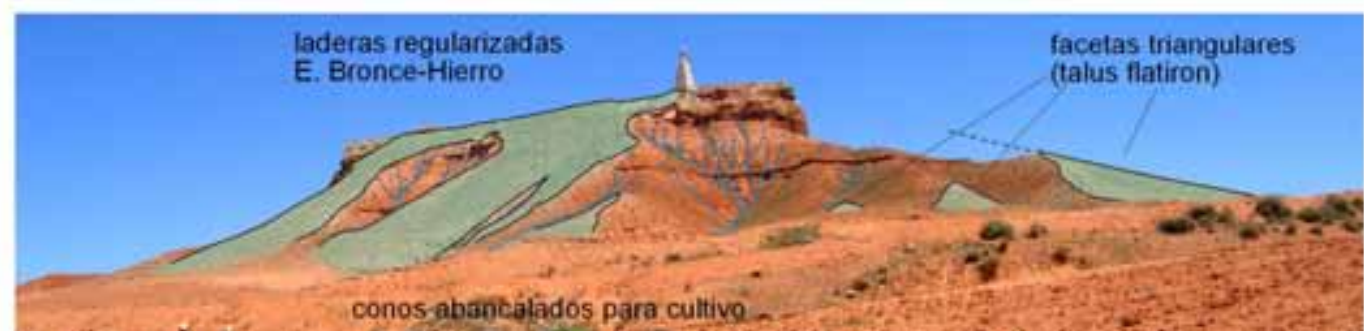

Figura 3. Esquema geomorfológico del cerro del castillo de Alfambra (Teruel) y vista panorámica de su ladera SW. Figure 3. Geomorphological scheme of the castle hill of Alfambra (Teruel) and panoramic view of its SW slope. 
climáticos (morfogénesis acelerada). La acción antrópica determina por tanto procesos de desestabilización de laderas casi equivalentes a los generados por climas secos (excepto en casos de repoblación forestal o medidas de restauración del paisaje, como casos excepcionales de reestabilización).

Los trabajos efectuados en el NE de España ponen de manifiesto que la sucesión de etapas alternantes de agradación/degradación en laderas se repite constantemente a lo largo de la evolución cuaternaria debido a los cambios climáticos, conservándose ya en antiguas regularizaciones en forma de facetas triangulares (talus flatirons), tanto del Pleistoceno superior como holocenas (Sancho et al., 1988; Gutiérrez et al., 1998). Entre esta últimas se han identificado dos etapas de agradación o estabilización ya en los primeros trabajos en la depresión de Alfambra-
Teruel, en la Cordillera Ibérica (Burillo et al., 1981a, $1981 b, 1983)$. Con vistas a conocer su generalización su estudio se fue extendiendo al sector central de la depresión del Ebro (Pellicer et al., 1986) y a la parte oriental de dicha cuenca (Peña-Monné et al., 1988; Peña-Monné and González, 1992; Peña-Monné and Rodanés, 1992; Peña-Monné et al., 1996; Sopena and Peña-Monné, 1998; González et al., 2005). Estos estudios confirman la mayor parte de los resultados iniciales y muestran el carácter generalizable de los mismos al NE de la Península lbérica y permiten establecer un modelo evolutivo para el Holoceno superior (Peña-Monné et al., 2005a). Finalmente, en los últimos años se ha realizado un estudio más detallado sobre la evolución de las laderas, la geoarqueología y el desarrollo de suelos en el curso bajo del río Huerva que completa la reconstrucción paleoambien-
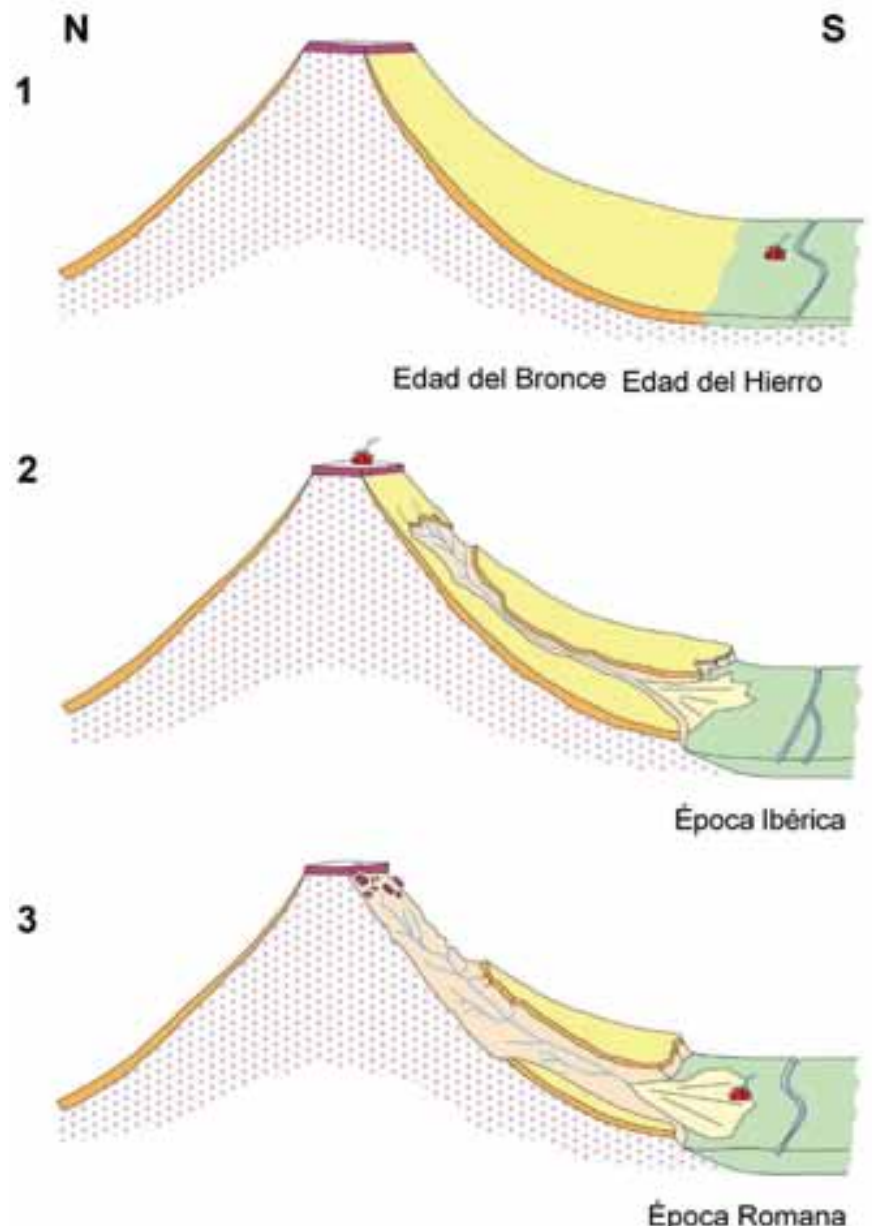

S
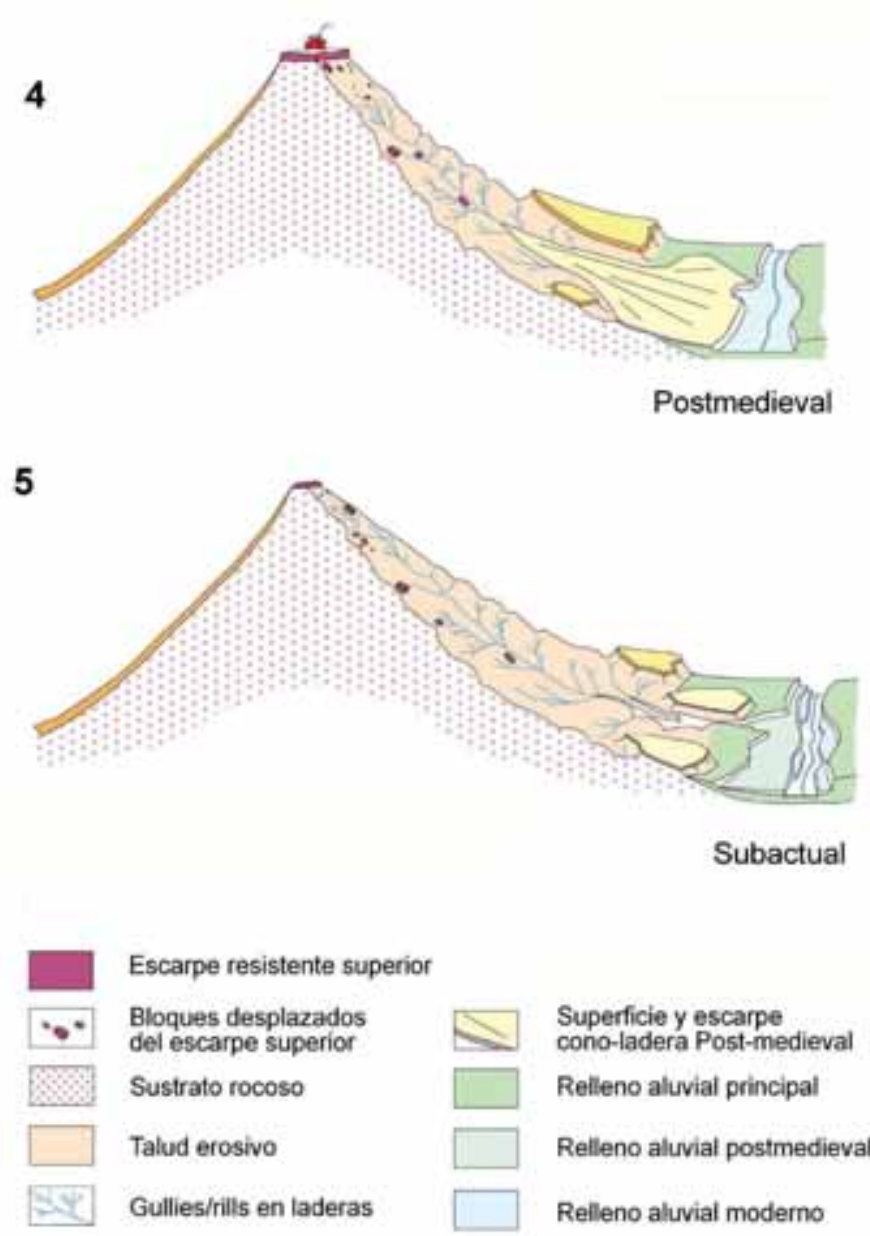

Figura 4. Modelo geoarqueológico mostrando las etapas evolutivas de las laderas del Baix Segre y Cinca (Lleida, Huesca) y su ocupación humana durante el Holoceno superior (sintetizado de Peña-Monné et al., 2005a).

Figure 4. Geoarchaeological model showing the evolutionary stages of the slopes of Baix Segre and Cinca (Lleida, Huesca) and its human occupation during the Late Holocene (synthesized from Peña-Monné et al., 2005a). 
Peña Monné, J.L., 2018. Geoarqueología aplicada a la reconstrucción paleoambiental:... Boletín Geológico y Minero, 129 (1/2): $285-303$

tal procedente de las laderas (Peña-Monné et al., 2011; Pérez-Lambán et al., 2014).

En el primer estudio realizado en estas laderas (Burillo et al., 1981a, 1981b) ya se definió la presencia en el paisaje de la Cordillera Ibérica (depresión de Alfambra-Teruel) de dos fases importantes de regularización de laderas debidas a procesos de estabilización por causas climáticas. De ellas, la más antigua (Fig. 4.1), definida entonces como de edad post-Edad del Bronce y pre-lbérica, es decir terminada en su forma final durante la $1^{\text {a }}$ Edad del Hierro, alcanza un fuerte grado de generalización, tanto en zonas altas de las Serranías Ibéricas orientales (1000-1600 m) como en la Depresión del Ebro, por debajo de los 200 $\mathrm{m}$. Actualmente se conoce que el inicio de esta gran etapa de regularización hay que situarla en el Calcolítico final (ca. $2300 \mathrm{BC}$ ) ya que en laderas de Peña Enroque, situadas la zona de Muel (Zaragoza), las cerámicas de esta época se sitúan en la base de la acumulación, fechas corroboradas además por dataciones ${ }^{14} \mathrm{C}$ (Pérez-Lambán et al., 2014).

Lo importante de estas laderas es que en la mayor parte de los casos contienen información de gran interés sobre la evolución de la ocupación humana en su entorno y especialmente en las partes altas de las mismas. En las zonas semiáridas de la depresión del Ebro en ocasiones estas laderas permanecen de forma residual tras desaparecer el relieve superior al que se adosaban y donde estaba el asentamiento original, siendo por tanto el único testimonio de un proceso de ocupación. Restos arqueológicos de las edades del Bronce y Hierro desplazados (fragmentos de cerámica, molinos, restos constructivos, huesos, etc.) además de otros restos in situ (como hogares, muros y otros testimonios de ocupación) aparecen intercalados en estas acumulaciones. Las características sedimentológicas y geomorfológicas del depósito indican que están generadas por procesos dominantemente de solifluxión debidos a una etapa de mayor humedad que la existente en la actualidad y que por su posición cronológica se corresponde paleoclimáticamente con la fase fría de la Edad del Hierro, cuyo pico de máximo frío y humedad se sitúa entre 800 y 650 BC (Van Geel et al., 1996; evento 2.8 de Bond et al., 1997, 2001) (ver figura 10, que sirve de síntesis final). Desde el punto de vista edafológico, estas condiciones de estabilidad favorecieron incluso la formación de un suelo, que normalmente apenas se ha conservado, aunque hay un testimonio excelente en las laderas de Peña Enroque, antes mencionadas, que ha sido denominado Paleosuelo IIIA (Pérez-Lambán et al., 2014). Este suelo todavía existía en época romana, ya que un pequeño hogar instalado en él ha dado una edad de 2152-1995 cal BP y se ha mantenido gracias a que fue cubierto por acumulaciones posteriores de ladera de la Pequeña Edad del Hielo (Fig. 5).

Estas características ambientales y la consiguiente dinámica de estabilización de laderas terminan a partir de la $2^{a}$ Edad del Hierro (Cultura Ibérica), ya que no contienen materiales de esta cronología. Por el contrario, a partir de estos momentos (épocas lbérica, Iberorromana, Romana) comienza un proceso de intensa erosión que da lugar a una red de incisiones, que en ocasiones alcanzarán a eliminar casi por completo la anterior forma regularizada. Esta desestabilización se genera en un lapso temporal breve, a partir de ca. $500 \mathrm{BC}$ y se prolonga hasta la actualidad, aunque con un periodo intermedio de interrupción en el que temporalmente se volverá a dinámicas nuevamente de estabilidad y regularización relacionadas con la Pequeña Edad del Hielo. En la génesis de esta ruptura brusca está por una parte un cambio ambiental, que es el paso del Subboreal al Subatlántico, de condiciones más secas y cálidas que el periodo anterior, pero además coincide con un aumento importante en la densidad de ocupación humana y en el incremento de la explotación de recursos mediante sobrepastoreo y agricultura que llevará consigo un alto grado de deforestación y desestabilización del sistema de laderas. Como consecuencia de ello, se formarán regueros y barrancos en las laderas, que irán ramificándose hasta llegar a generar en algunos casos redes de cárcavas (badlands), cuando las litologías basales lo favorezcan. El testimonio y cronología de esta etapa está registrado en los fondos de los valles inmediatos en forma de rellenos y conos aluviales. Este proceso erosivo, debido a su mayor humedad ambiental, será menor en las laderas orientadas al N y NW, mientras que las $\mathrm{S}$ y SE alcanzará su máximo desarrollo (Figs. 3, 4.2, 4.3).

Como hemos indicado, la segunda fase de regularización de laderas se produce en la Pequeña Edad del Hielo. Lo más habitual es que estas nuevas laderas se formen en las áreas en las que ha desaparecido ya la anterior ladera tras un largo proceso denudativo (desde ca. $500 \mathrm{BC}$ hasta los siglos XV-XVI AD, aproximadamente). Ni su extensión ni desarrollo acumulativo es comparable a la anterior fase, ya que su periodo de formación es más corto y las condiciones ambientales de humedad necesarias tampoco alcanzaron tales características. Sin embargo, ha quedado su huella geomorfológica en forma de regularizaciones de poco espesor en el interior de las anteriores cárcavas o pequeños conos cuyos ápices ascienden ladera arriba (Fig. 4.4). De forma excepcional pueden formar laderas extensas al pie de las plataformas terciarias (muelas) de la cuenca del Ebro. Estas laderas se las había considerado como "post-Medievales" en 

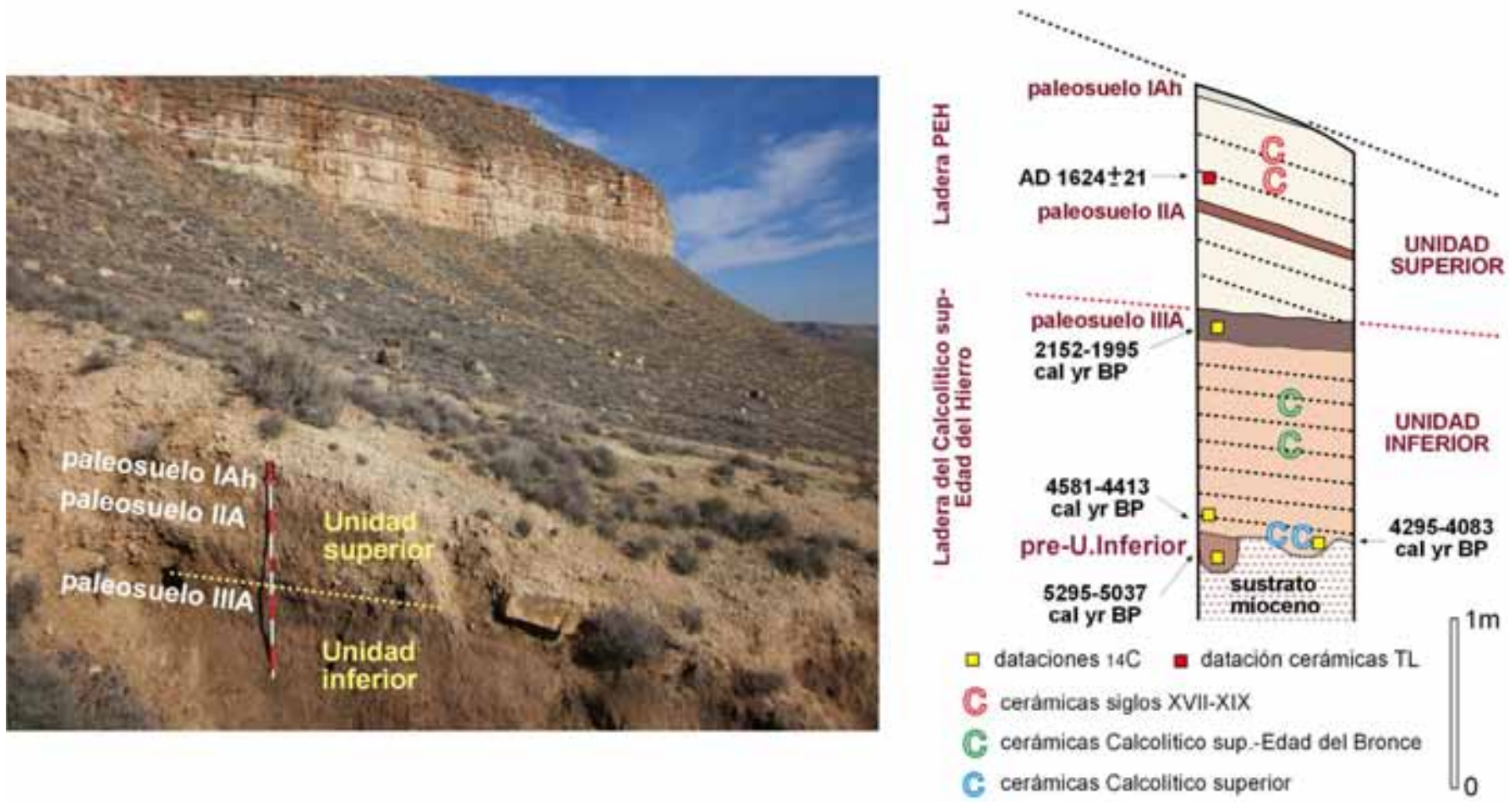

Figura 5. Perfil sintético y fotografía de las laderas de Peña Enroque (Muel, Zaragoza). Se han representado las Unidades diferenciadas, los niveles de paleosuelos, las dataciones $\left(\mathrm{TL},{ }^{14} \mathrm{C}\right.$ ) y los restos arqueológicos (basado en Pérez-Lambán et al., 2014).

Figure 5. Synthetic profile and photography of the slopes of Peña Enroque (Muel, Zaragoza). Main units, paleosol levels, dating (TL, ${ }^{14} \mathrm{C}$ ), and archaeological remains are represented (based on Pérez-Lambán et al., 2014).

términos generales al no existir dataciones precisas (Peña-Monné and González, 1992; Peña-Monné et al., 1996). Sin embargo, en el antes mencionado relieve de Peña Enroque, estas laderas se superponen a las de la fase de la Edad del Bronce-Hierro (Fig. 5), cubriendo el paleosuelo IIIA mencionado y mostrando una importante acumulación con dos fases internas separadas por estabilizaciones y desarrollo de suelos incipientes (paleosuelos IIA y IAh), con una datación de $1624+/-21$ d.C. (fecha obtenida mediante datacion TL de una cerámica incluida en el depósito) (Pérez-Lambán et al., 2014). Tras la PEH esta etapa también ha sido afectada por la reanudación de la incisión (Fig. 4.5; Fig. 10c).

\section{Los abrigos rocosos y su evolución}

Un caso especial de registro geoarqueológico en laderas es la reconstrucción de los procesos de configuración y evolución de abrigos rocosos en áreas de alternancia de rocas resistentes con formaciones más blandas. Son abrigos formadas por un voladizo rocoso y un entrante más o menos profundo abierto en las rocas blandas inferiores, que han servido para la ocu- pación humana en diferentes momentos culturales. Este tipo de abrigos es muy abundante en todo el NE de España, aunque adquiere una especial significación en las formaciones molásicas del piedemonte pirenaico catalán de la depresión del Ebro así como en otros ámbito similares de la Cadena Costera Catalana. Los estudios geomorfológicos de la evolución de este tipo de formas y su repercusión en la prospección geoarqueológica se han centrado en el valle medio y bajo del río Segre y de algunos de sus afluentes (Peña-Monné and González, 1999; PeñaMonné and Vázquez, 2000; Peña-Monné et al., 2002, 2005a, 2011a, 2011b). El inicio de los mismos estuvo en relación con las dificultades generadas por la prospección de laderas recubiertas de bloques rocosos (esencialmente areniscas y microconglomerados oligocenos) para la identificación de materiales arqueológicos ubicados fuera de contexto y con una apreciable mezcla de épocas y estilos.

En el Segre medio, en términos generales, en la Edad del Bronce inicial existían numerosos abrigos o voladizos ya configurados, muchos de ellos incluso más antiguos, pero en esa época adquieren una utilización masiva (Fig. 6a). Como se ha señalado anteriormente en las laderas, es un periodo coincidente 


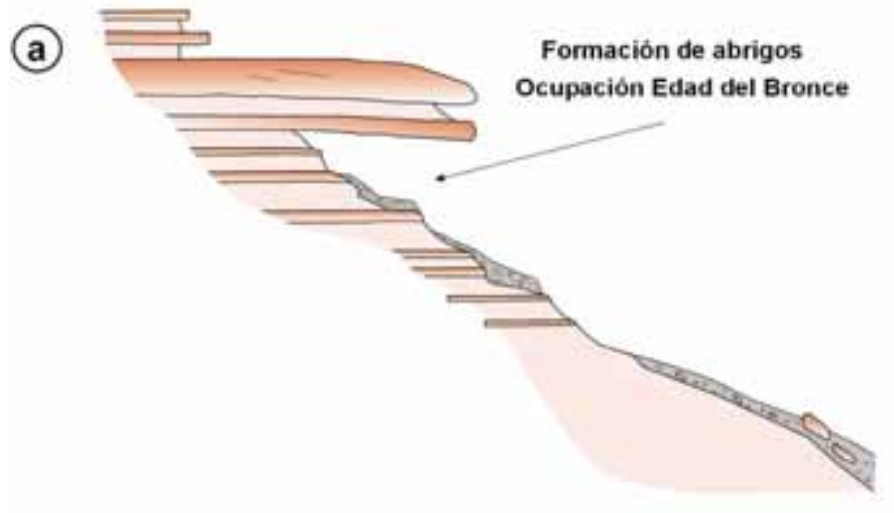

(c)

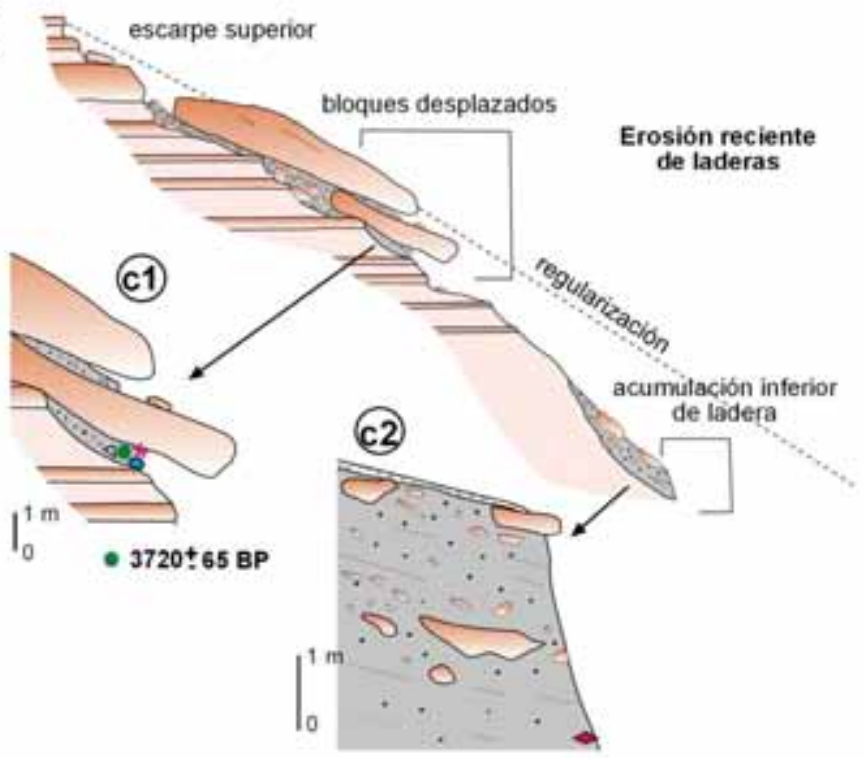

(b)

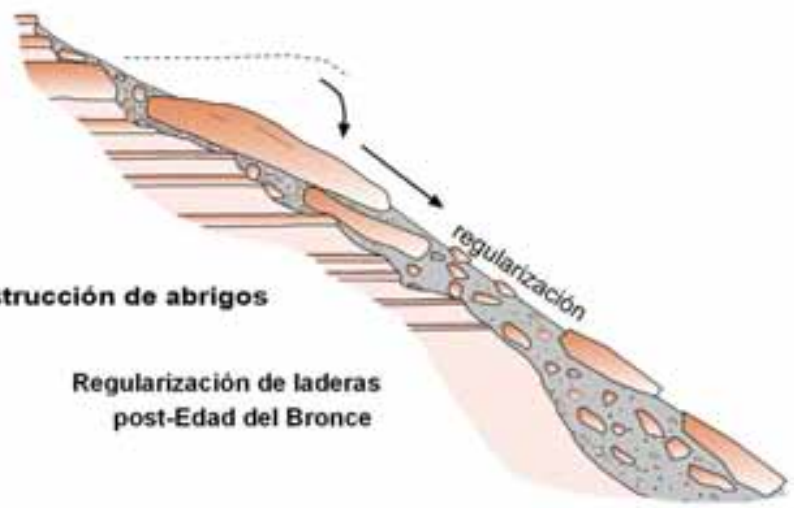

Destrucción de abrigos

Regularización de laderas post-Edad del Bronce

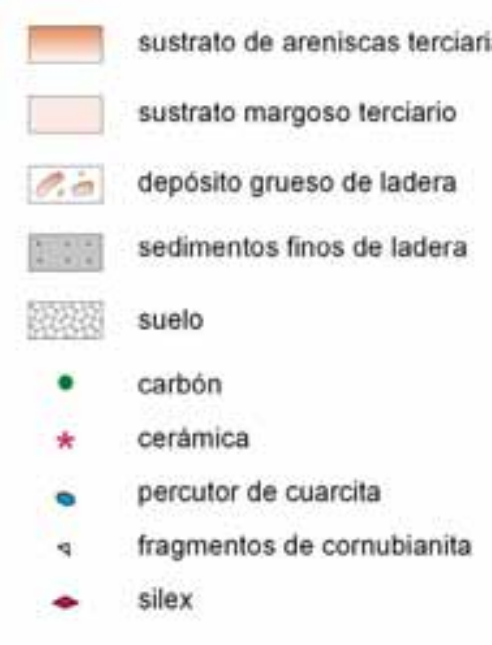

Figura 6. Evolución de un abrigo o balma de areniscas oligocenas en el valle medio del río Segre (Lleida). a) Formación de abrigos con anterioridad a la Edad del Bronce y ocupación humana durante dicha época. b) Caída del voladizo y desplazamiento ladera abajo finalizando con una regularización general del depósito (Edad del Hierro). c) Situación actual tras el desmantelamiento de las laderas por la erosión, reduciéndose la información de la antigua ocupación del abrigo a dos puntos de la ladera: bajo los bloques desplazados (c1) y en la parte baja (c2), de los que se muestran detalles (basado en Peña-Monné and González,1999).

Figure 6. Evolution of a rock shelter made over Oligocene sandstone in the middle valley of Segre River (Lleida). a) Rock shelter formation previous to Bronze Age, human occupation during that epoch. b) Fall of the cantilever and movement downslope ending with a general regularization of the deposit (Iron Age). c) Present situation after the slope erosion. The information of the rock shelter occupation is reduced to two points of the slope: under the displaced blocks (cl) and in the lower part (c2), details are shown (based on Peña-Monné and González, 1999).

con una mayor humedad ambiental que culmina en el evento 2,8 de Bond et al. (1997; 2001), es decir en torno a 2800 BP. Los procesos de profundización de los abrigos comenzarán a producir desplomes de los voladizos antes de la Edad del Hierro ya que apenas hay materiales de esta época. Estos desplomes presentan diferentes tipos de caída y posterior desplazamiento ladera abajo por procesos esencialmente de solifluxión que dejaron sin protección la estratigrafía del interior de los abrigos. En definitiva, bloques rocosos y materiales arqueológicos se dispersaron y mezclaron ladera abajo debido a la erosión consi- guiente. La configuración final de la ladera es la misma que se ha descrito como general para las laderas en la Edad del Hierro: una regularización que sella todo el proceso de destrucción de abrigos (Fig. 6b). En numerosos lugares se conserva esta forma regularizada sin posterior evolución, siendo visibles los restos arqueológicos sólo en cortes artificiales (caminos, carreteras) (Fig. 6c2). Pero lo más habitual es que la erosión reciente resultante de la fuerte deforestación haya generado la desaparición de la forma estabilizada y la profundización de regueros ( $r i l l s$ ) que se han llevado los materiales más finos, dejando en evi- 
a

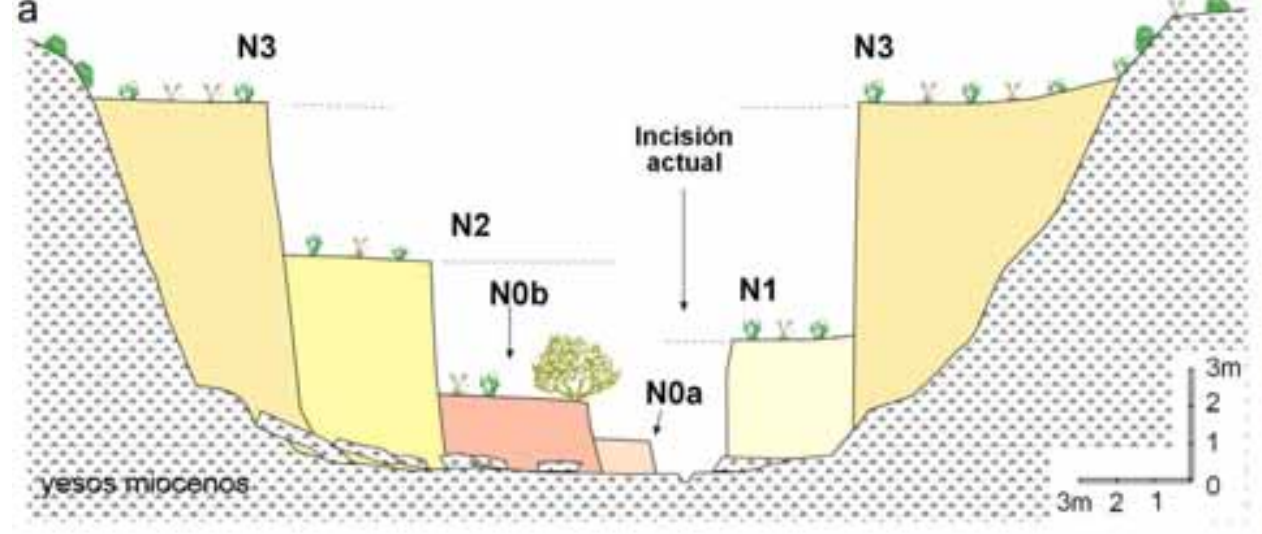

N3 Fase principal de relleno (Neolitico-Romano tardio)
N2 Fase relleno post-Medieval (PEH 1)

NOb NOa Fases relleno úttimas décadas
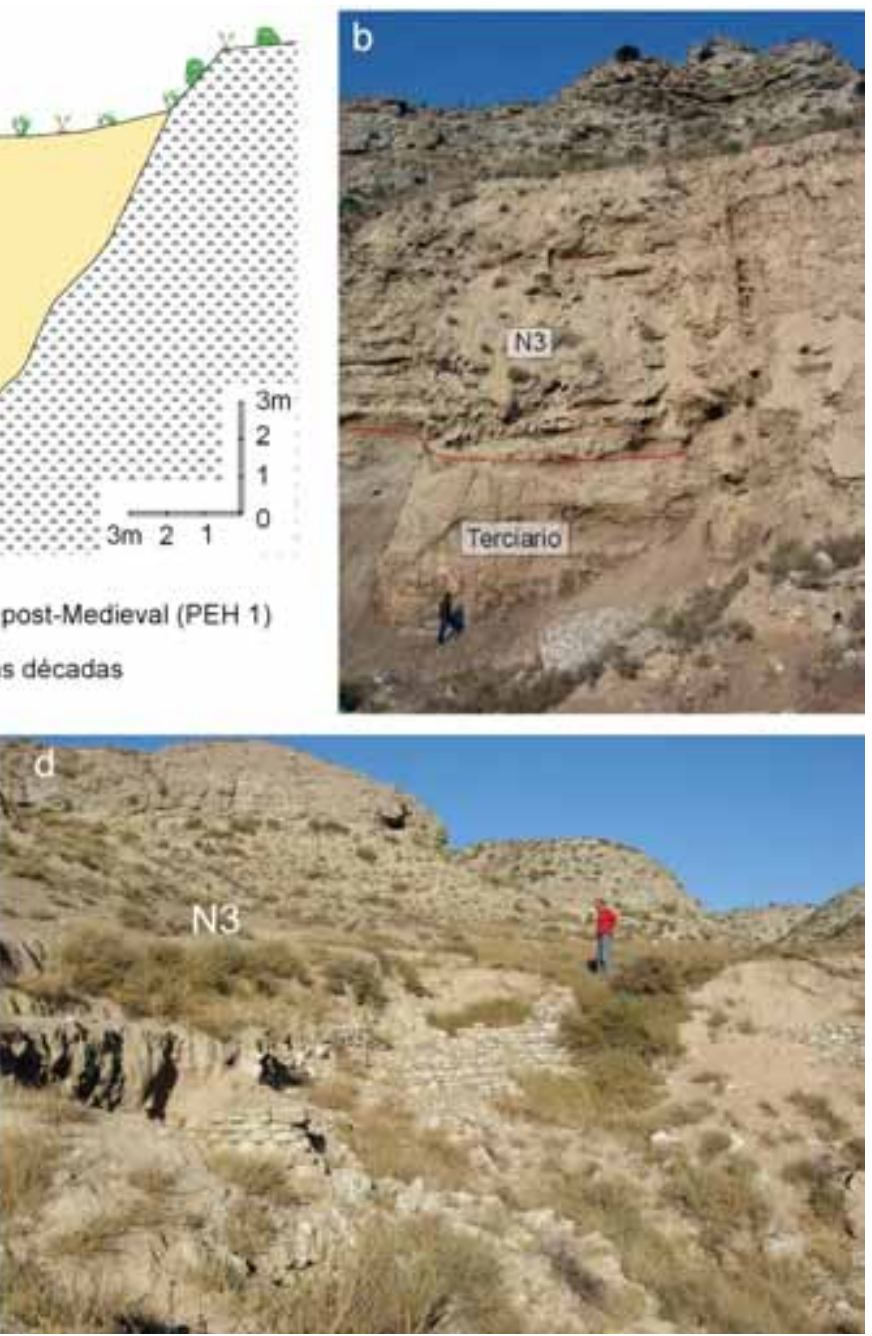

Figura 7. Rellenos holocenos, procesos de incisión y geoarqueología en el sector central de la cuenca del Ebro. a) Corte transversal detallado de la val de la Morera (valle del Huerva, Zaragoza), con las etapas N3, N2 y N1 así como las fases recientes de la segunda mitad del siglo XX (NOb, N0a) (Peña-Monné et al., 2013). b) Profunda incisión de la val de la Virgen sobre el nivel N3 y el sustrato mioceno, al N del río Ebro cerca de Alagón (Zaragoza). c) Niveles N3 y N2 en la val de la Virgen. d) Casas de época ibérica exhumadas por la incisión sobre el relleno N3 de una val afluente del río Ginel, cerca de Mediana de Aragón (Zaragoza).

Figure 7. Holocene infills, incision processes and geoarchaeology in the central part of the Ebro Basin. a) Detailed transversal cut of the Val de la Morera (Huerva Valley, Zaragoza), with N3, N2, and N1 stages as well as recent phases of the second half of S XX (NOb, NOa) (Peña-Monné et al., 2013). b) Val de la Virgen deep incision made over N3 level and the Miocene substrate, N of Ebro River, close to Alagón (Zaragoza). c) N3 and N2 levels in the Val de la Virgen. d) Iberian houses exhumed by the incision over N3 infill of one val tributary of Ginel River, close to Mediana de Aragón (Zaragoza).

dencia los antiguos voladizos caídos (Fig. 6c) y quedando concentraciones de restos arqueológicos de tamaño medio y grande a modo de pavimentos debido al lavado selectivo. Por otra parte, es posible encontrar situaciones de mejor preservación geoarqueológica, como en el caso de materiales protegidos por bloques y más cercanos a la situación original, como en el ejemplo de la Figura 6c1 correspondiente al sector de Anya-Torreblanca (Lleida), en el que hay algunos útiles, cerámicas y restos de carbón que permiten dataciones del asentamiento bajo la visera ori- ginal (Peña-Monné et al., 2002). En la figura 10d se han representado las etapas evolutivas de abrigos en su posición cronológica.

\section{La información geoarqueológica aportada por los rellenos de valle $y$ conos aluviales}

Frente a la evolución geomorfológica de los sistemas fluviales durante el Pleistoceno, caracterizada por la formación de terrazas cuaternarias en los ríos princi- 
pales, la evolución holocena es mejor conocida por los rellenos que presentan los pequeños cursos afluentes. Ello es debido a que las terrazas más recientes de los ríos han estado sujetas a continuos procesos de erosión y reposición de sedimentos, que apenas dejan posibilidades de obtener etapas bien diferenciadas. Por ello, el tipo de acumulaciones fluviales analizadas con enfoque geoarqueológico son siempre valles secundarios que se caracterizan por su fondo plano, que son conocidas en Aragón como vales. El mejor desarrollo de estas vales se ha producido en el sector central del valle del Ebro y sobre litologías yesíferas y arcillosas miocenas. De tal forma que la mayor parte de estos estudios se han efectuado en un radio de unos $25 \mathrm{~km}$ de la ciudad de Zaragoza, que es lo que abarca aproximadamente el área de afloramiento de dichos yesos. También las Bardenas Reales de Navarra presentan modelados de una cierta similitud, en un contexto litológico, geomorfológico y ambiental diferente (Sancho et al., 2007a, 2008).

Estas zonas fueron muy ocupadas en la Antigüedad, pero los continuos procesos erosivos derivados de cambios climáticos y la acción antrópica han determinado la desaparición de gran parte de los datos de los asentamientos. Tal es así que los registros geoarqueológicos aportan la mejor información sobre los momentos de ocupación desde el Neolítico hasta época Romana. Por ello se iniciaron trabajos en esta línea por van Zuidam (1975), Burillo et al. (1985a), Soriano (1989), Soriano and Calvo (1987), Peña-Monné et al. (1991, 1993), Arauzo and Gutiérrez (1994) que aportaron los primeros datos sobre estos rellenos aluviales. En una segunda fase, los trabajos efectuados en las vales afluentes del río Huerva (Peña-Monné et al., 1996, 1998, 2001, 2004; Ángeles et al., 2004), afluente del río Ebro, y en otros tributarios de este último río permitieron completar las dataciones de las etapas diferenciadas y una interpretación más completa de la secuencia evolutiva holocena y su relación con la ocupación humana de estos espacios (Peña-Monné,1996; Constante et al., 2006, 2009, 2010, 2011). En la zona NE de Zaragoza, Sancho et al. (2007b) también analizan la depresión de Valsalada (Leciñena) desde un punto de vista geoarqueológico.

Algunos de estos valles conservan restos de niveles más antiguos que los rellenos holocenos que vamos a describir y así aparecen en algunos trabajos de Peña-Monné et al. (1996, 2004), Constante et al. (2010), diferenciando hasta tres terrazas antiguas (N6, N5, N4), seguramente todas pleistocenas. Presentan un dispositivo escalonado y están compuestas de clastos calizos procedentes del arrastre desde las laderas de las muelas calizas miocenas que forman sus cabeceras. Sin embargo, los niveles de terrazas holocenas presentan depósitos dominantemente finos, especialmente el nivel que hemos denominado N3 en diferentes publicaciones. Esta acumulación marca un importante ruptura en la evolución sedimentaria de las vales ya que se trata de un importante registro geoarqueológico que podemos considerar de origen antrópico en cuanto al desencadenamiento de los procesos de erosión que lo han generado (Constante and Peña-Monné, 2009a, 2009b; PeñaMonné et al., 2014; Peña-Monné and SampietroVattuone, 2014).

Se han diferenciado tres niveles de agradación holocena separados por fases de incisión, que se han denominado N3, N2 y N1, a los que se añaden otros dos niveles más recientes (segunda mitad siglo $X X$ ) denominados N0a y NOb (Figs. 7a, 7c). El nivel N3 se inició a partir de un valle en $\mathrm{V}$ formado a inicios del Holoceno por incisión sobre las terrazas antiguas siguiendo el proceso iniciado en los ríos principales (terrazas del río Huerva, por ejemplo). El proceso de relleno del valle N3 se inició en fechas variables según las zonas, aunque la mayor parte presentan dataciones en la base de la acumulación en torno a 6000-7000 BP, es decir situadas en el Óptimo Climático y en el periodo cultural del Neolítico (Fig. 8). Se trata de un primer aporte detrítico relativamente grueso, que hemos denominado "Nivel detrítico basal" y constituye el subnivel A de la Figura 8.1 y 8.4. El depósito continuó para generar un segundo conjunto sedimentario (B) a partir de ca. $3500-2500$ $\mathrm{BP}$, que abarca las Edades del Bronce y Hierro, conteniendo en su interior cerámicas de esas épocas arrastradas conjuntamente con el material detrítico. Estos depósitos $\mathrm{B}$ son correlativos de la fase de estabilización de laderas de la que ya se ha hablado anteriormente, que acaba configurada en torno al final de la $1^{\text {a }}$ Edad del Hierro. Los subniveles posteriores C y D se acumularon en un periodo más breve de tiempo, desde 2200-2000 BP hasta el siglo IV d-C. (época Romana Tardía), es decir en unos 400-600 años y constituyen la parte superior del depósito, básicamente limoso, que es consecuencia de un incremento en las tasas de erosión en las laderas debido a la sobrexplotación antrópica. En sus términos más altos (D) este depósito llega a recubrir todas las villas romanas allí instaladas con 0,5-1 $\mathrm{m}$ de limos (Fig. 8.4), así como las zonas bajas de los yacimientos arqueológicos (Fig. 7d). El resultado final es un valle ampliamente colmatado de sedimentos dando una morfología plana, alcanzando 10 a $15 \mathrm{~m}$ de espesor total (Figs. 7b, 7c).

El relleno N3 de las vales se continúa hacia la llanura de inundación del valle principal formando 


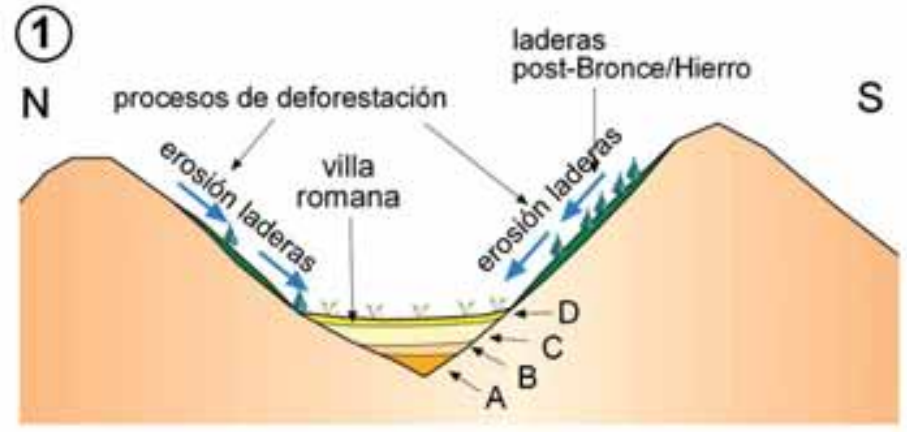

Fases de relleno (nivel principal) de valles de fondo plano (vales)

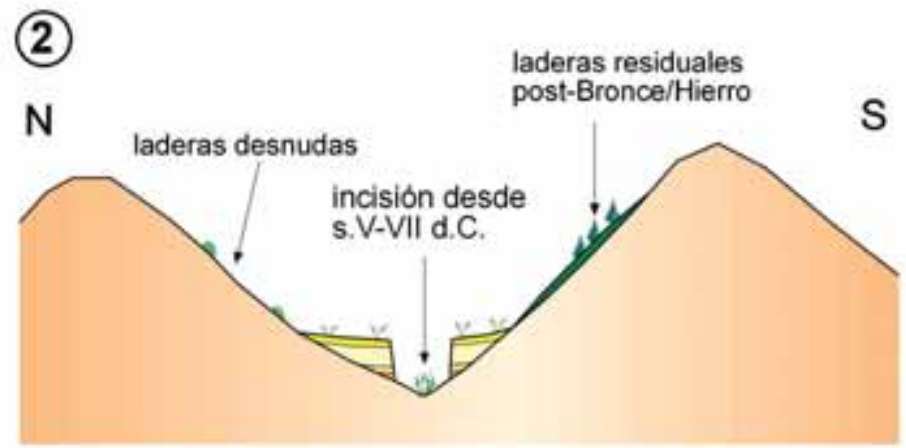

Proceso de incisión principal
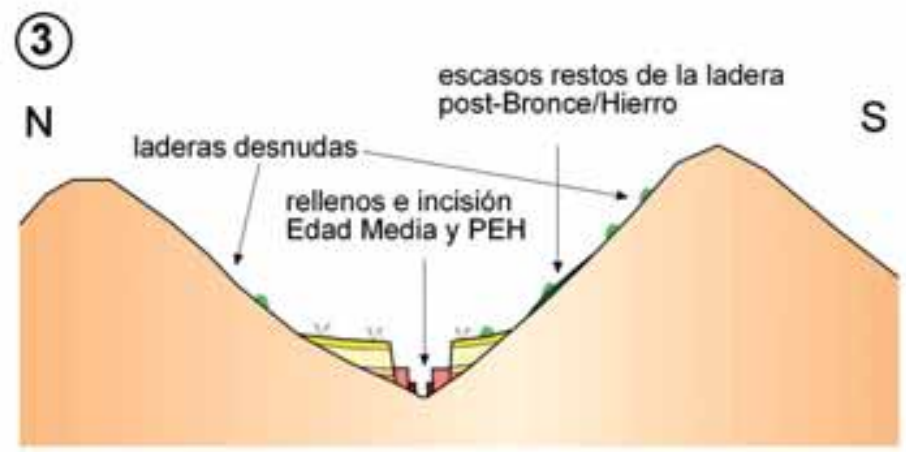

Fases de incisión y relleno recientes en las vales

(4)

Limos

Post-Romano tardio (s.II-IV dC)

Limos intermedios procedentes de la erosión de los suelos y laderas

Epoca Romana

Epoca Ibero-romana

Capas detriticas paso Suboreal al Subatlántico Edades Bronce/Hierro

Nivel detritico basal

Optimo Atlántico Neolitico
B escarpe del Ebro incremento erosion $3500-2500$ B.P:

yalle del Huerva incremento erosion $2200-2000$ B.P.

A

inicio erosión 6000-7000 B.P.

Figura 8. Esquemas evolutivos de la formación de los rellenos holocenos de los valles de fondo plano (vales) en el sector central de la depresión del Ebro. 1) acumulación del nivel principal de relleno (N3) por erosión de las laderas, diferenciándose cuatro fases internas (A, B, C, D) que abarcan desde el Neolítico hasta época Romana tardía. 2) incisión post-Romana (a partir de los siglos V-VII d.C.). 3) Etapas acumulativas y de incisión medievales y de la PEH (N2, N1). 4) Perfil sintético del nivel N3, con información de su cronología y relación con etapas culturales (basado en Peña-Monné et al., 2004, 2013).

Figure 8. Evolutionary schemes of the formation of Holocene infills in the flat valleys (vales) in the central area of the Ebro Depression. 1) main level accumulation (N3) by slope erosion, with internal phases $(A, B, C, D)$ that covers from Neolithic to Late Roman epoch. 2) After Roman incision (V-VII centuries). 3) Cumulative and incisional stages Middle Age and LIA (N2, N1). 4) Synthetic profile of N3 level, with chronological information and relationships with cultural stages (based on Peña-Monné et al., 2004, 2013).

amplios conos aluviales. Los sedimentos son dominantemente limos y arenas, cuyo espesor disminuye desde la zona proximal de salida del valle hasta el enlace con el fondo aluvial del río principal. En las vales situadas al $\mathrm{N}$ del río Ebro (vales de Alfocea, de la Virgen) se aprecia la misma cronología que la propuesta para los valles, superponiéndose sucesivos conos desde el Neolítico hasta época Romana (Fig. 9a) e incluso continuando hasta incluir los conos post-medievales a techo de la acumulación (Peña- 
a

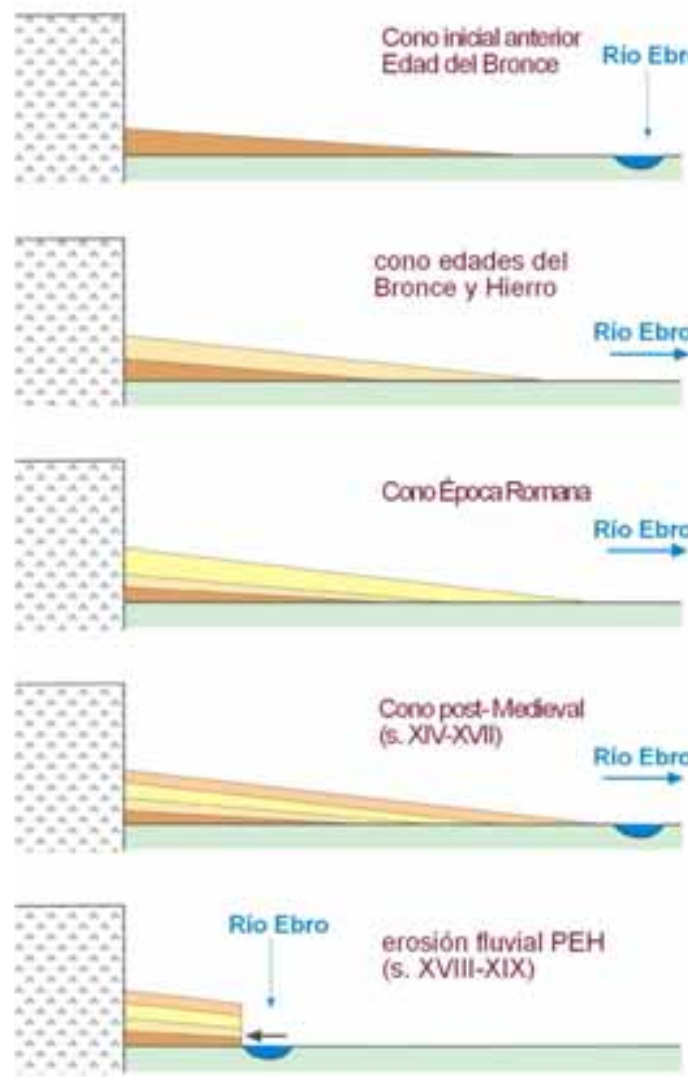

D CONO DEL SISALLAR

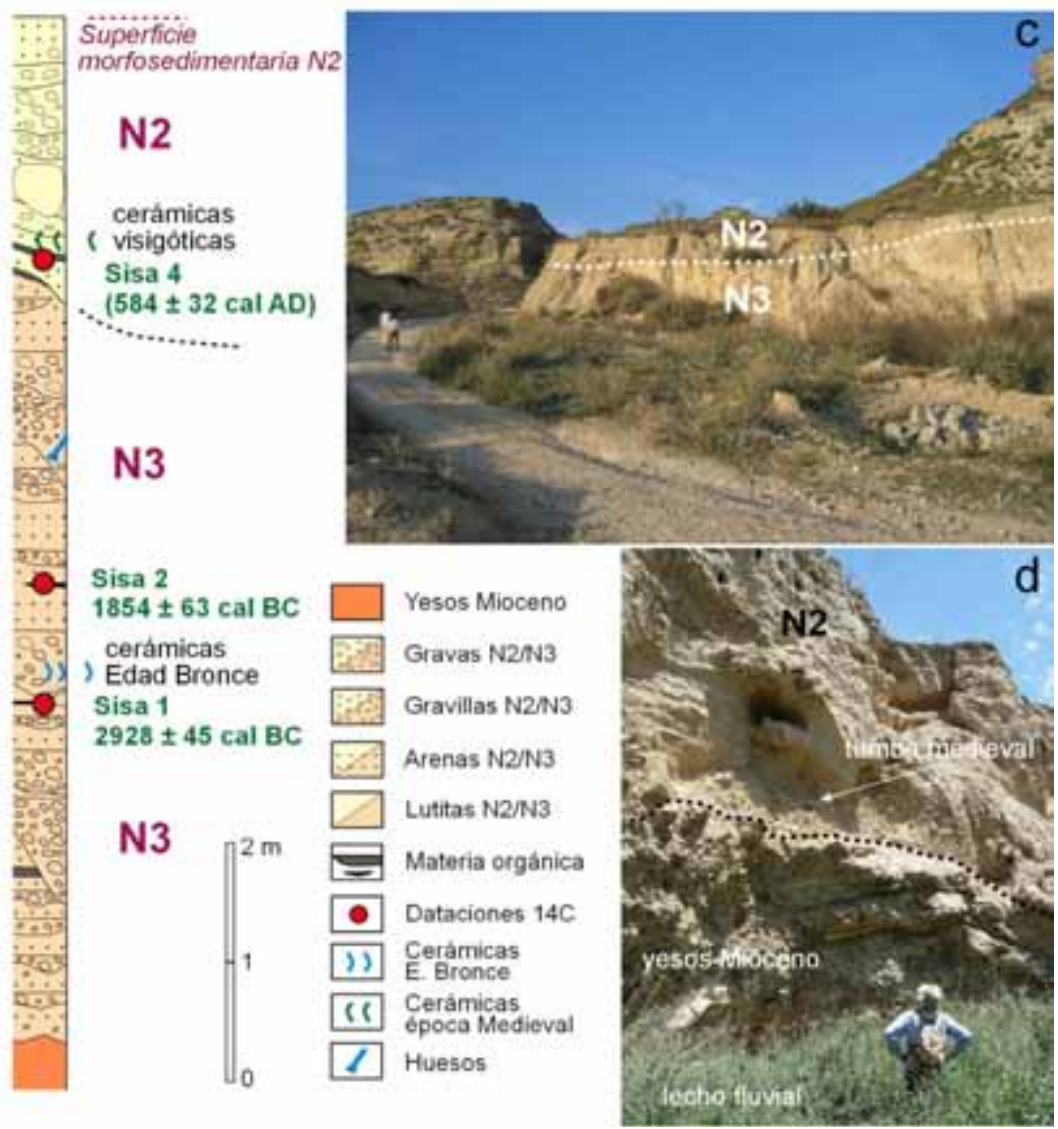

Figura 9. Conos holocenos y geoarqueología en el sector central del valle del Ebro. a) Evolución holocena de los conos de las vales del escarpe de yesos de Alagón-Juslibol (Zaragoza), al $\mathrm{N}$ del río Ebro. El río se mantuvo alejado de este margen fluvial desde el Neolítico hasta la PEH, en que erosionó la mayor parte de los conos, dejando algunos cortes residuales (Constante et al., 2011). b) Perfil del cono del Sisallar, compuesto por la superposición del nivel N2 sobre el conjunto N3, con abundante información geoarqueológica. c) Cono de la val de Alfocea, con los dos niveles superpuestos (N3 y N2). d) Cono N2 de la val de la Virgen, depositado directamente sobre el sustrato rocoso y conteniendo tumbas medievales en su interior.

Figure 9. Holocene cones and geoarchaeology in the central sector of Ebro Valley. a) Holocene evolution of the cones of the vales in the gypsum scarp of Alagón-Juslibol (Zaragoza), $N$ of Ebro River. The river was far away from this fluvial margin from Neolithic to LIA, when most of the cones were eroded, leaving some residual profiles (Constante et al., 2011). b) Cone profile from Sisallar composed of the superposition of $\mathrm{N} 2$ level over the set N3, with abundant geoarchaeological information. $c$ ) Val de Alfocea cone, with the two overlying levels (N2 and N3). d) N2 cone from Val de la Virgen, lying over the bedrock and with Middle Age tombs inside.

Monné et al., 2009; Constante et al., 2011). Es interesante observar que la construcción de estos conos sobre la Ilanura aluvial del Ebro se ha mantenido sin rupturas desde el Neolítico hasta la Pequeña Edad del Hielo. En ese momento, el río Ebro se desplazó hacia el $\mathrm{N}$ del valle y erosionó los frentes haciéndolos retroceder hasta la zona proximal (Fig. 9a), de manera que los cortes observables presentan gran espesor, como en el cono del Sisallar (Fig. 9b), cuya cronología se ha establecido mediante dataciones ${ }^{14} \mathrm{C}$ y con cerámicas de la Edad del Bronce para el nivel N3 y con cerámicas visigóticas y dataciones para la etapa N2 o postMedieval (Constante et al., 2011). La misma superpo- sición se aprecia en el cono de Alfocea (Fig. 9c). En otras zonas, como el valle del Huerva, los conos N3 aparecen bien individualizados y la etapa de conos N2 aparece en posición encajada formando un cono independiente.

Se puede considerar a N3 como una acumulación de génesis antrópica resultado de la morfogénesis acelerada iniciada en el Neolítico y con fuerte incremento a partir de época iberorromana. Esta es la fase más importante de acumulación holocena, abarcando desde final del Boreal hasta pleno Subatlántico, coincidiendo en este último periodo con condiciones secas y cálidas. Su máximo acumulativo -dado su ori- 


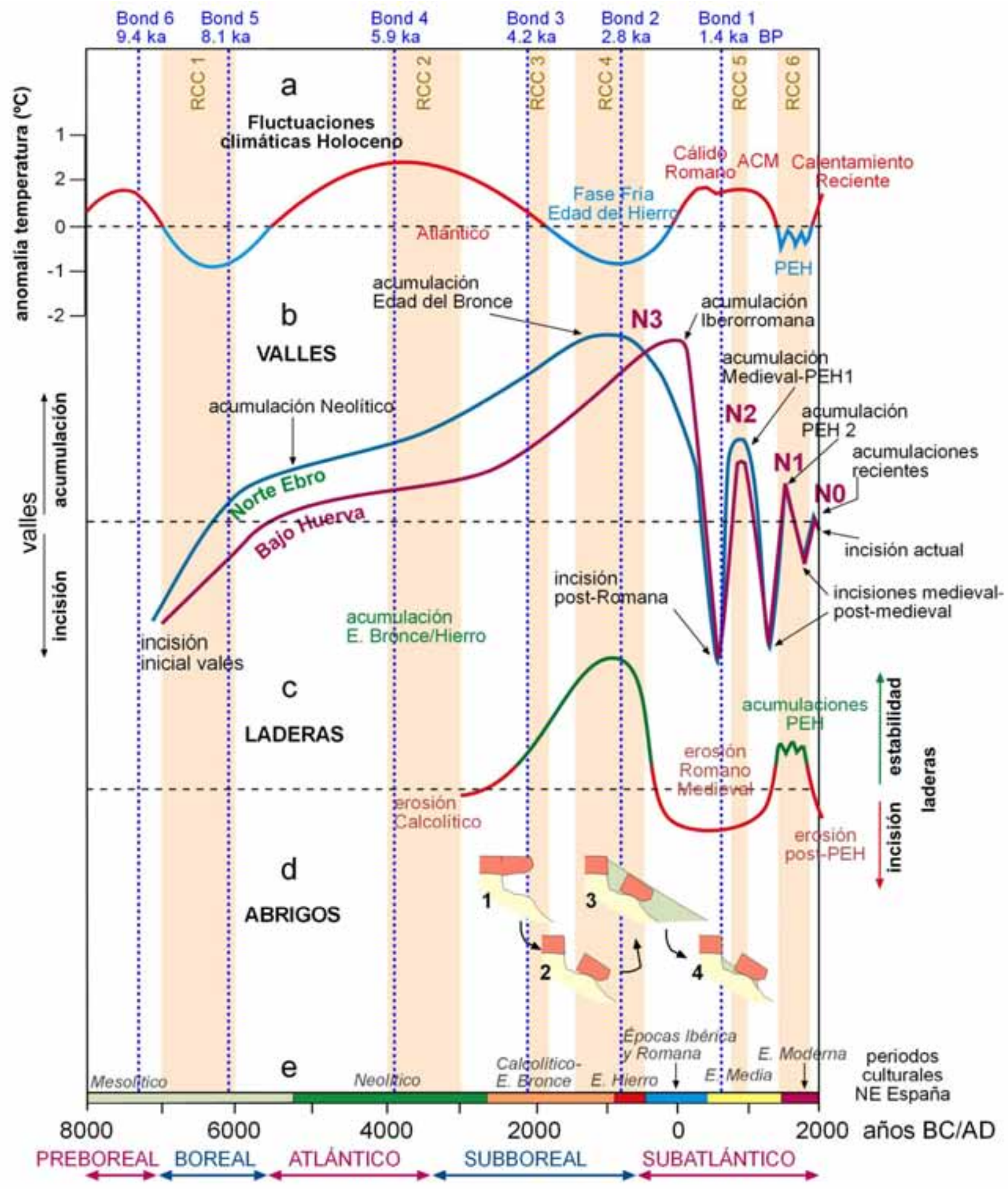

Rapid Climatic Changes

Mayewski et al., 2004

Bond events

Bond et al., 1997

Figura 10. Gráfica representando la evolución temporal (a) y cultural (e) durante las fases de agradación y degradación en los valles (b) y laderas (c) y en la evolución de abrigos prehistóricos en el NE de España (basados en Peña Monné et al., 2014, Pérez-Lambán et al., 2014). Figure 10. Graphic representing the temporal evolution (a) and cultural evolution (e) during the aggradation and degradation phases in the valleys (b) and slopes (c) and the evolution of Prehistoric rock shelters in NE Spain (based on Peña-Monné et al., 2014, Pérez-Lambán et al., 2014). 
gen antrópico- puede variar según las zonas (PeñaMonné et al., 2014), como reflejan las gráficas de la Fig. 10b.

A partir del siglo $\mathrm{V}$ la terraza N3 comienza a ser incidida por los propios cursos fluviales. El proceso se genera básicamente por sufosión (piping) hasta abrir un surco que profundiza hasta alcanzar el sustrato rocoso infrayacente e incluso superarlo (Figs. 7b, 8.2), exponiendo a la vista los perfiles de N3. Este proceso de incisión está muy generalizado en las vales del norte del Ebro, mientras que las del río Huerva sólo están afectadas un pequeño porcentaje de ellas, destacando las vales de las Lenas y de la Morera (Peña-Monné et al., 2004).

En el interior de esta incisión y sólo allí se formarán los nuevos niveles N2 y N1 (Fig. 8.3), ambos generados en dos etapas de la Pequeña Edad del Hielo, que también han sido registradas en las laderas, como ya se ha indicado, en el sector de Peña Enroque (Muel, Zaragoza). Son acumulaciones de menor espesor que las de N3 y sus depósitos presentan un dominio de gravas semirrodadas de litología calcárea procedentes de la cabecera de la cuenca, es decir nada que ver con las formaciones limosas del nivel principal N3. En algunas vales, como la de la Virgen, al N del Ebro, la terraza N2 alcanza cierta extensión y genera un amplio cono aluvial, que se apoya directamente sobre el sustrato de yesos (Fig. 9d), sin embargo lo habitual es que el nivel N2 forme una estrecha terraza, que además ha sido erosionada a tramos posteriormente. Por su parte, N1 es un depósito muy inestable, ya que al quedar muy cerca de la incisión funcional, en crecidas esporádicas ha llegado a desaparecer de algunas vales. Como hemos indicado, incluso en la incisión actual que corta los tres niveles holocenos hay dos acumulaciones más, bien visibles en el barranco de la Morera, cerca de María de Huerva, con fechas entre los años 1950 a 1979 (Saz et al., 2004) y debidas a tormentas puntuales. El origen climático de las acumulaciones N2 y N1 queda patente por sus características sedimentarias coincidiendo las dataciones existentes en situarlas en fluctuaciones de la Pequeña Edad del Hielo con eventos de lluvias intensas (Fig. 10b).

\section{Conclusiones}

En regiones semiáridas, como gran parte del NE de España, numerosos yacimientos arqueológicos han sufrido notables modificaciones por procesos de erosión y acumulación, por lo que la realización de estudios geoarqueológicos es una tarea imprescindible para reconstruir su evolución.
El estudio de las etapas de agradación/incisión en laderas, valles y conos aluviales, entendidos como un sistema de funcionamiento acoplado, permite el establecimiento de una evolución conjunta marcada por las fases de estabilización y desestibilización en las laderas y fases de acumulación e incisión en los valles y conos en forma de terrazas. Las fluctuaciones climáticas holocenas son las causantes de muchos de estos procesos, mientras que otros están favorecidos e incluso provocados por la intervención humana. En este último caso estaría la fase de incisión en laderas desde época Iberorromana hasta la actualidad, sólo interrumpida por la estabilización de causa climática de la Pequeña Edad del Hielo. También la formación de la acumulación N3 en valles y conos se interpreta como consecuencia de esa misma fase de desestabilización antrópica de las laderas, generando un registro geoarqueológico muy importante (N3), ya que incluye muchos restos de yacimientos desaparecidos en la actualidad.

En definitiva, el establecimiento de modelos evolutivos para diferentes ambientes geomorfológicos, basados en una metodología geoarqueológica, es decisivo para realizar labores de prospección efectivas y completar la información sobre la evolución del paisaje, los sistemas de poblamiento y la explotación de los recursos naturales en diferentes épocas del pasado.

\section{Agradecimientos}

Este trabajo sintetiza una de las líneas de investigación del Grupo Consolidado de Investigación Paleoambientes del Cuaternario PALEOQ del Gobierno de Aragón, enmarcado en el IUCA (Instituto de Ciencias Ambientales de Aragón) y se ha efectuado con ayuda del Proyecto HAR2012-36967 del Ministerio de Economía y Competitividad. Agradezco a la Dra. M.M. Sampietro Vattuone su ayuda en la preparación del artículo.

\section{Referencias}

Angeles, G., J.L. Peña-Monné, J.L. and Longares, L.A. 2004. Cartografías de reconstrucción paleoambiental y riesgo geomorfológico del Holoceno superior en el valle del Río Huerva (Depresión del Ebro). In: Peña-Monné, J.L., Longares, L.A. and Sánchez, M. (eds.), Geografía Física de Aragón. Aspectos generales y temáticos. Univ. Zaragoza e Inst. Fernando el Católico, 303-314.

Arauzo, T. and Gutiérrez, M. 1994. Evolución de los valles de fondo plano del centro de la Depresión del Ebro. In: Arnáez, J., García-Ruiz, J.M. and Gómez, A. (eds.), 
Geomorfología en España, III Reunión de Geomorfología, Logroño, I, 277-290.

Bintliff, J. 1976. Sediments and settlement in Southern Greece. In: Davidson, D.J. and Shackley, M.L. (eds.), Geoarchaeology, Duckworth, 267-275.

Bond, G., Kromer, B., Beer, J., Muscheler, R., Evans, M.N., Showers, W., Hoffmann, S., Lotti-Bond, R., Hajdas, I. and Bonani, G. 2001. Persistent solar influence on North Atlantic climate during the Holocene. Science, 278, 12571266.

Bond, G., Showers,W., Cheseby, M., Lotti, R., Almasi, P., de Menocal, P., Priore, P., Cullen, H., Hajdas, I. and Bonani, G. 1997. A pervasive millennial-scale cycle in north Atlantic Holocene and glacial climates. Science, 278, 1257-1266.

Burillo, F., Gutiérrez, M. and Peña-Monné, J.L. 1981a. El cerro del castillo de Alfambra (Teruel). Estudio interdisciplinar de Geomofología y Arqueología. Kalathos, 1, 1-60.

Burillo, F., Gutiérrez, M. and Peña-Monné, J.L. 1981b. Las vertientes del cerro del castillo de Alfambra (Teruel). $V$ Reunión Nacional Grupo Español de Trabajo del Cuaternario, 231- 244.

Burillo, F., Gutiérrez, M. and Peña-Monné, J.L. 1983. La Geoarqueología como ciencia auxiliar. Una aplicación a la Cordillera Ibérica Turolense. Revista de Arqueología, $26,6-13$.

Burillo, F., Gutiérrez, M. and Peña Monné, J.L. 1985a. Las acumulaciones holocenas y su datación arqueológica en Mediana de Aragón (Zaragoza). Cuadernos de Investigación Geográfica, 11, 193-207.

Burillo, F., Gutiérrez, M. and Peña-Monné, J.L. 1985b. Datación arqueológica de deformaciones tectónicas en vertientes holocenas de Sierra Palomera (Cordillera Ibérica Centroriental). Actas I Reuniao do Quaternario Ibérico, II , 355-366.

Burillo, F., Gutiérrez, M., Peña-Monné, J.L. and Sancho, C. 1986. Geomorphological processes as indicators of climatic changes during the Holocene in the North-East Spain. In: López-Vera, A. (ed.): Quaternary Climate in Western Mediterranea, 31-44.

Burillo, F. and Peña Monné, J.L. 1984a. Modificaciones por factores geomorfológicos en el tamaño y ubicación de los asentamientos primitivos. Arqueología Espacial, 1, 91-105.

Burillo, F. and Peña-Monné, J.L. 1984b. Clima, geomorfología y ocupación humana. Introducción a un planteamiento metodológico. Actas I Jornadas de Metodología de Investigación Prehistórica. Soria 1981, 91-102.

Burillo, F., Peña-Monné, J.L. and Picazo, J. 1985c. Acción del arroyamiento en yacimientos de conjuntos líticos y modelos de reconstrucción. Aplicación en Mora de Rubielos. Actas XVII Congreso Nacional de Arqueología. Logroño 1983, 81-88.

Brückner, H. 1986. Man's impact on the evolution of the physical environment in the Mediterranean region in historical times. GeoJournal, 13 (1), 7-17.

Butzer, K.W. 1980. Holocene alluvial sequences: problems in dating and correlation. In: Cullingford, R.A., Davidson, D.A. and Lewin, J. (eds.), Timescales in Geomorphology. Wiley, 131-142.
Constante, A., Dossche, R., Peña-Monné, J.L., Sancho, C. and de Dapper, M. 2006. Dinámica morfosedimentaria holocena en laderas y valles del escarpe de AlfoceaJuslibol (sector central de la Depresión del Ebro). In: Pérez Alberti, A. and López Bedoya, J. (eds.), Geomorfología y territorio, Santiago de Compostela, 979-992.

Constante, A., Dossche, R., Peña-Monné, J.L., Sancho, C. and de Dapper, M. 2009. Holocene evolution and geoarchaeology in the Ebro valley around Zaragoza (Northern Spain). In: De Dapper, M., Vermeulen, F., Deprez, S. and Taelman, D. (eds.), Ol man river. Geo-archaeological aspects of rivers and river plains, Academia Press, Ghent, 241-256.

Constante, A. and Peña-Monné, J.L. 2009a. Human-induced erosion and sedimentation during the Holocene in the central Ebro depresión, Spain. In: Romero, A., Belmonte, F., Alonso, F. and López-Bermúdez, F (eds.), Advances in studies on desertification, Universidad de Murcia, 207210.

Constante, A. and Peña-Monné, J.L. 2009b. Particularidades sedimentarias en el Holoceno del sector central de la Depresión del Ebro. In: Boski, T, Moura, D. and Gomes, A. (eds.), El futuro del ambiente de la Península lbérica: lecciones del pasado geológico reciente. VII Reuniao do Quaternário Ibérico. Faro (Portugal), 250-254.

Constante, A., Peña-Monné, J.L. and Muñoz, A. 2010. Alluvial geoarchaeology of an ephemeral stream: Implications for Holocene landscape change in the Central part of the Ebro Depression, Northeast Spain. Geoarchaeology, 25, 475-496. <<http://dx.doi.org/ 10.1002/gea.2031>>

Constante, A., Peña-Monné, J.L., Muñoz, A. and Picazo, J. 2011.Climate and anthropogenic factors affecting alluvial fan development during the Late Holocene in the Central Ebro valley, Northeast Spain. The Holocene, 21, 275-286.

Davidson, A.D., 1980. Erosion in Greece during the first and second millennia BC. In: Cuilingford, R.A., Davidson, R.A. and Levin, J. (eds.), Timescales in Geomorphology. John Wiley and Sons, New York 143-158.

González-Pérez, J.R., Peña-Monné, J.L. and RodríguezDuque, J.I. 2005. El tossal de Moradilla (Lleida) en el marco evolutivo del Holoceno superior de la Depresión del Ebro. In: Santonja, M, Pérez González, A. and Machado, M.J. (eds.), Geoarqueología y Patrimonio en la Península Ibérica y el entorno mediterráneo, 383-394.

González-Sampériz, P. and Sopena, M.C. 2002. Recent Holocene palaeoenvironmental evolution in the central Ebro Basin (NE Spain). Quaternary International, 93-94, 177-190.

Gutiérrez, M. and Peña-Monné, J.L. 1992. Evolución climática y geomorfológica del Holoceno superior (Cordillera Ibérica, Depresión del Ebro y Prepirineo). In: Cearreta, A. and Ugarte, F.M. (eds.), The Late Quaternary in the Western Pyrenean Region, 109-124.

Gutiérrez-Elorza, M. and Peña-Monné, J.L. 1998. Geomorphology and Late Holocene Climatic Change in Northeastern Spain. Geomorphology, 23, 205-217.

Gutiérrez, M., Arauzo, T., Sancho, C. and Peña-Monné, J.L. 1998. Evolution and paleoclimatic meaning of the talus 
Peña Monné, J.L., 2018. Geoarqueología aplicada a la reconstrucción paleoambiental:... Boletín Geológico y Minero, 129 (1/2): $285-303$

flatirons in the Ebro Basin, northeast Spain. In: Alsharhan et al. (eds.), Quaternary Deserts and Climatic Change, Balkema, 593-599.

Mayewski, P.A., Rohling, E.E, Stager, J.C., Karlén, W., Maasch, K.A., Meeker, L.D., Meyerson, E.A., Gasse, F., Kreveld, S., Holmgren, K., Lee-Thorp, J., Rosqvist, G., Rack, F., Staubwasser, M., Schneider, R.R. and Steig E.J. 2004. Holocene climate variability. Quaternary Research, 62, 243-255.

Pellicer, F., Peña-Monné, J.L. and Ibáñez, M.J. 1986. Estudio geomorfológico del yacimiento de Burrén y Burrena (Depresión del Ebro). Génesis del relieve y evolución holocena. Estudios Homenaje Prof. A. Beltrán, Zaragoza, 33-45.

Peña-Monné, J.L. 1983 a. La Conca de Tremp y las Sierras Prepirenaicas comprendidas entre los ríos Segre $y$ Noguera Ribagorzana, Inst. Estudios Ilerdensese, Lleida, $373 \mathrm{pp}$.

Peña-Monné, J.L. 1983 b. Dinámica reciente de vertientes en el valle medio del Segre (zona Anya-Artesa de Segre, prov. de Lérida). Actas VIII Coloquio de Geografía, Barcelona, 123-130.

Peña-Monné, J.L. 1996. Los valles holocenos del escarpe de yesos de Juslibol (sector central de la Depresión del Ebro). Aspectos geomorfológicos y geoarqueológicos. Arqueología Espacial, 15, 83-102.

Peña-Monné, J.L., Chueca, J., Julián, A. and Echeverría, M.T. 1996. Reconstrucciones paleoambientales en el sector central de la Depresión del Ebro a partir de rellenos de valle y conos aluviales. In: Pérez-Alberti, A., Martini, P., Chesworth, W. and Martínez-Cortizas, A., (eds.), Dinámica y evolución de medios cuaternarios, 291-307.

Peña-Monné, J.L., Echeverría, M.T., Chueca, J. and Julián, A. 2001. Processus géomorphologiques d accumulation et incision pendant I Antiquité Classique et ses rapport avec I activité humaine et les changements climatiques holocènes dans la vallée de la Huerva (Bassin de I Ebre, Espagne). In: Vermeulen, F. and de Dapper, M. (eds.), Geoarchaeology of the Landscapes of Classical Antiquity, Ed. Peeters. Leuven (Belgium), 151-159.

Peña-Monné, J.L., Echeverría, M.T., Petit-Maire, N. and Lafont, R. 1993. Cronología e interpretación de las acumulaciones holocenas de la Val de las Lenas (Depresión del Ebro, Zaragoza). Geographicalia, 30, 321-332.

Peña-Monné, J.L. and González, J.R. 1992. Hipótesis evolutiva de los cambios en la dinámica geomorfológica del Baix Cinca y Segre (Depresión del Ebro) durante el Pleistoceno superior-Holoceno a partir de los datos geoarqueológicos. Cuaternario y Geomorfología, 6, 103-110.

Peña-Monné, J.L. and González, J.A. 1999. Evolució dels abrics de gres a la vall mitjana del riu Segre (sector oriental de la Depressió de I Ebre). Geoarqueologia $i$ Quaternari litoral, Memorial M.P. Fumanal, Universidad de Valencia, Valencia, 217-227.

Peña-Monné, J.L., González, J.R. and Rodríguez, J.I. 1988. Estudi geoarqueológic del Tossal de Moradilla (Lleida). Recerques Terres de Ponent, 9, 31-41.

Peña-Monné, J.L., González, J.R. and Rodríguez, J.I. 1996. Paleoambientes y evolución geomorfológica en yacimientos arqueológicos del sector oriental de la depre- sión del Ebro durante el Holoceno superior. In: PérezAlberti, A., Martini, P., Cherworth, W. and MartínezCortizas, A. (eds.), Dinámica y evolución de medios cuaternarios, Xunta de Galicia, Santiago de Compostela, 63-80.

Peña-Monné, J.L., Julián, A. and Chueca, J. 1991. Séquences évolutives des accumulations holocènes à la Hoya de Huesca dans le contexte général du Bassin de I Ebre (Espagne). Physio-Géo, 22-23, 55-60.

Peña-Monné, J.L., Julián, A., Chueca, J. and Echeverría, M.T. 1998. Los estudios geoarqueológicos en la reconstrucción del paisaje. Su aplicación en el valle bajo del río Huerva. Arqueología Espacial, 19-20, 169-183.

Peña-Monné, J.L., Julián, A., Chueca, J., Echeverría, M.T. and Ángeles, G. 2004. Etapas de evolución holocena en el valle del río Huerva: Geomorfología y Geoarqueología. In: Peña-Monné, J.L., Longares, L.A. and Sánchez, M. (eds.), Geografía Física de Aragón. Aspectos generales y temáticos, Universidad Zaragoza e Institución Fernando el Católico, 289-302.

Peña-Monné, J.L., Pérez-Lambán, F., Picazo, J. and Fanlo, J. 2011a. Secuencias paleoclimáticas y geoarqueología en laderas holocenas del valle del río Huerva (Depresión del Ebro). In: Turu, V. and Constante, A. (eds.), EI Cuaternario en España y áreas afines. Avances en 2011. XIII Reunión Nacional del Cuaternario, Andorra la Vella, 217-219.

Peña-Monné, J.L. and Rodanés, J.M. 1992. Evolución geomorfológica y ocupación humana en el cerro de Masada de Ratón (Baix Cinca, prov. de Huesca). Cuaternario y Geomorfología, 6, 81-89.

Peña-Monné, J.L., Rubio, V. and González, J.R. 2002. Cambios dinámicos en laderas holocenas del valle del Segre (Depresión del Ebro). In: Peña-Monné, J.L. and Longares, L.A. (eds.), Aportaciones Geográficas en memoria del Prof. L. Miguel Yetano Ruiz, Universidad de Zaragoza, Zaragoza, 421-432.

Peña-Monné J.L., Rubio, V. and González, J.R. $2005 a$. Aplicación de modelos geomorfológicos evolutivos al estudio de yacimientos arqueológicos en medios semiáridos (Depresión del Ebro, España). X Coloquio Ibérico de Geografía. A Geografia ibérica no cotexto europeo. Évora, 15 p. Edición CD http://www.apgeo.pt/files/ docs/CD_X_Coloquio_lberico_Geografia/pdfs/076.pdf

Peña-Monné, J.L, Rubio, V., González, J.R., Rodanés, J.V., Picazo, J., Medina, J., Vázquez, M.P., SampietroVattuone, M.M. and Pérez-Lambán, F. 2014a. Geoarchaeology of defensive moats: its importante for site localization, evoluction and formation process reconstruction of archaeological sites in NE Spain. Journal of Archaeological Science, 50, 383-393. http://dx.doi.org/10.1016/j.jas.2014.07.026

Peña-Monné, J.L., Rubio, V., Longares-Aladrén, L.A., Gutiérrez-González, F.J., Pérez-Lambán, F. and LalienaCorbera, C. 2014b. The geomorphological context of Medieval Juslibol Castle in the middle reaches of the River Ebro, Spain. Geoarchaeology, 29, 448-461. http://dx.doi.org/10.1002/gea.21495

Peña-Monné, J.L. and Sampietro Vattuone, M.M. 2014. Geoarchaeological and paleoenvironmental reconstruc- 
tion through evolutionary models: Dryland applications. European Geologist Magazine, 38, 40-44.

Peña-Monné, J.L., Sancho, C., Muñoz, A. and Constante, A. 2014. Clima y hombre en la evolución de las vales del sector central de la Depresión del Ebro durante el Holoceno superior. In: Arnáez, J., González, P., Lasanta, T. and Valero, B.L. (eds.), Geoecología, cambio ambiental y paisaje: homenaje al Profesor José María García Ruiz. Instituto Pirenaico de Ecología (CSIC)-Universidad de La Rioja, 91-102.

Peña-Monné, J.L., Sancho, C. and Rubio, V. 2005b. Aportaciones desde la geomorfología y la geoarqueología al conocimiento de los paleoambientes holocenos del NE de España. X Coloquio Ibérico de Geografía. A Geografia ibérica no cotexto europeo. Évora, 15 p. Edición CD. http://www.apgeo.pt/files/docs/CD_X_ Coloquio_lberico_Geografia/pdfs/077.pdf

Peña-Monné, J.L., Sopena, M.C., Rubio, V., Constante, A. and González, J.R. 2011. Registros geoarqueológicos residuales y reconstrucción de yacimientos en medios semiáridos del NE de España. In:Turu, V. and Constante, A. (ed.) El Cuaternario en España y áreas afines. Avances en 2011. XIII Reunión Nacional del Cuaternario, Andorra la Vella, 257-260.

Peña-Monné, J.L. and Vázquez, M.P. 2000. Estudio geoarqueológico del yacimiento de Puig Pelegrí (Segriá, Lleida). Rev. d'Arqueologia de Ponent ,10, 277-292.

Pérez Lambán, F., Peña Monné, J.L., Fanlo, J., Picazo, J.V., Badia, D., Rubio. V., García-Jiménez, R. and SampietroVattuone, M.M. 2014. Paleoenvironmental and geoarchaeological reconstruction from late Holocene slope records (Lower Huerva Valley, Ebro Basin, NE Spain). Quaternary Research, 8, 1-14.

Pope, K.O. and van Andel, T.H.1984. Late Quaternary alluviation and soil formation in the southern Argolid: its history, causes and archaeological implications. Journal of Archaeological Science, 11, 281-306.

Rubio, V., Peña-Monné, J.L. and González, J.R. 2006. EI impacto en el paisaje de los fosos de época prehistórica en el NE de España y su reconocimiento con criterios geomorfológicos. III Congr. Internacional sobre Fortificaciones "Paisaje y Fortificación", Alcalá de Guadaira (Spain), 55-68.

Sancho, C., Benito, G., Peña-Monné, J.L., Longares, L.A., McDonald, E., Rhodres, E. and Saz, M.A. 2007a. Actividad aluvial durante la Pequeña Edad del Hielo en Bardenas Reales de Navarra. Geogaceta, 42, 111-114.
Sancho, C., Gutiérrez, M., Peña Monné, J.L. and Burillo, F. 1988. A quantitative approach to scarp retreat starting from triangular slope facets (Central Ebro Basin, Spain). In: Harvey, A.M. and Sala, M. (eds.), Geomorphic processes In Environments With Strong Seasonals Contrasts. II: Geomorphic Systems. Catena Suppl. 13, 139-146.

Sancho, C., Peña-Monné, J.L., Muñoz, A., Benito, G., McDonald, E., Rhodes, E.J. and Longares, L.A. 2008. Holocene alluvial morphopedosedimentary record and environmental changes in the Bardenas Reales Natural Park (NE Spain). Catena, 73, 225-238.

Sancho, C., Muñoz, A, Peña-Monné, J.L. and González, P. 2007b. Evolución morfosedimentaria del sistema aluvial de Valsalada (Leciñena, Los Monegros) durante el Pleistoceno superior-Holoceno: aproximación paleoambiental. Geogaceta, 41, 199-202.

Saz, M.A., Peña, J.L., Longares, L.A., Creus, J. and GonzálezHidalgo, J.C. 2004. Evolución reciente de fondos de valle holocenos en el sector central del valle del Ebro a partir de series de crecimiento radial de Tamarix canariensis. In: Peña-Monné, J.L., Longares, L.A. and Sánchez, M. (eds.), Geografía Física de Aragón. Aspectos generales y temáticos. Universidad Zaragoza e Institución Fernando el Católico, Zaragoza, 315-328.

Sopena, M.C. and Peña, J.L. 1998. Evolución del paisaje del Holoceno superior en el valle del Cinca, sector de Binaced (Huesca). Arqueología Espacial, 19-20, 185-197.

Soriano, M.A. 1989. Infilled valleys in the Central Ebro Basin (Spain). Catena, 16, 357-367.

Soriano, M.A. and Calvo, J.M. 1987. Características, datación y evolución de los valles de fondo plano de las inmediaciones de Zaragoza. Cuaternario $y$ Geomorfología, 1, 283-293.

Van Geel, B., Buurman, J. and Waterbolk, H. 1996. Archaeological and palaeoecological indications of an abrupt climate change in the Netherlands, and evidence for climatological teleconnections around 2650 BP. Journal of Quaternary Science, 11 (6), 451-460.

Van Zuidam, R.A. 1975. Geomorphology and Archaeology. Evidences of interrelation at historical sites in the Zaragoza region, Spain. Zeitscrift fur Geomorphologie, 19, 319-328.

Vita-Finzi, C., 1969. The Mediterranean Valleys. Cambridge Univ. Press, $140 \mathrm{pp}$.

Recibido: marzo 2015

Revisado: abril 2015

Aceptado: abril 2015

Publicado: marzo 2018 
